OPEN ACCESS

Edited by:

Simone Brogi,

University of Pisa, Italy

Reviewed by:

Keiichi Fukuda,

School of Medicine, Keio University, Japan

In-Hyun Park

Yale University, United States Andrew Laslett,

Biomedical Manufacturing,

Commonwealth Scientific and Industrial Research Organisation,

Australia

*Correspondence:

Mi-Ok Lee

molee@kribb.re.kr

Hyuk-Jin Cha

hjcha93@snu.ac.kr

Seok-Ho Kim

ksh3410@cha.ac.kr

tThese authors have contributed equally to this work

Specialty section: This article was submitted to Medicinal and Pharmaceutical Chemistry,

a section of the journal Frontiers in Chemistry

Received: 21 December 2018 Accepted: 12 April 2019 Published: 16 May 2019

Citation:

Go Y-H, Lim C, Jeong H-C Kwon O-S, Chung S, Lee H, Kim W, Suh Y-G, Son WS, Lee M-O, Cha H-J and Kim S-H (2019) Structure-Activity

Relationship Analysis of YM155 for Inducing Selective Cell Death of Human Pluripotent Stem Cells.

Front. Chem. 7:298.

doi: 10.3389/fchem.2019.00298

\section{Structure-Activity Relationship Analysis of YM155 for Inducing Selective Cell Death of Human Pluripotent Stem Cells}

\author{
Young-Hyun Go ${ }^{1+}$, Changjin Lim ${ }^{2 t}$, Ho-Chang Jeong ${ }^{1+}$, Ok-Seon Kwon ${ }^{3}$, \\ Sungkyun Chung ${ }^{2}$, Haeseung Lee ${ }^{4}$, Wankyu Kim ${ }^{4}$, Young-Ger Suh ${ }^{2}$, Woo Sung Son ${ }^{2}$, \\ Mi-Ok Lee ${ }^{3 *}$, Hyuk-Jin Cha ${ }^{5,6 *}$ and Seok-Ho Kim ${ }^{2 *}$ \\ ${ }^{1}$ Department of Life Sciences, College of Natural Sciences, Sogang University, Seoul, South Korea, ${ }^{2}$ Department of \\ Pharmacy, College of Pharmacy and Institute of Pharmaceutical Sciences, CHA University, Pochen-si, South Korea, ${ }^{3}$ Stem \\ Cell Convergence Research Center, Korea Research Institute of Bioscience and Biotechnology, Daejeon, South Korea, \\ ${ }^{4}$ Department of Life Science, Ewha Womans University, Seoul, South Korea, ${ }^{5}$ College of Pharmacy, Seoul National University, \\ Seoul, South Korea, ${ }^{6}$ Research Institute of Pharmaceutical Sciences, Seoul National University, Seoul, South Korea
}

Despite great potential for regenerative medicine, the high tumorigenic potential of human pluripotent stem cells (hPSCs) to form undesirable teratoma is an important technical hurdle preventing safe cell therapy. Various small molecules that induce the complete elimination of undifferentiated hPSCs, referred to as "stemotoxics," have been developed to facilitate tumor-free cell therapy, including the Survivin inhibitor YM155. In the present work, based on the chemical structure of YM155, total 26 analogs were synthesized and tested for stemotoxic activity toward human embryonic stem cells (hESCs) and induced PSCs (iPSCs). We found that a hydrogen bond acceptor in the pyrazine ring of YM155 derivatives is critical for stemotoxic activity, which is completely lost in hESCs lacking SLC35F2, which encodes a solute carrier protein. These results suggest that hydrogen bonding interactions between the nitrogens of the pyrazine ring and the SLC35F2 protein are critical for entry of YM155 into hPSCs, and hence stemotoxic activity.

Keywords: stemotoxics, naphthoquinone imidazolium, SAR (structure-activity relationship), human pluripotent stem cells, teratoma, YM155

\section{INTRODUCTION}

Human pluripotent stem cells (hPSCs), such as human embryonic stem cells (hESCs) and induced PSCs (iPSCs), have been actively studied due to their pluripotency, which allows them to produce all cell types in the human body and renders them a promising source for stem cell-based regenerative therapy (Trounson and Dewitt, 2016). Recent success in clinical trials of hESC-derived retinal pigment epithelial cells, for reversing loss of vision in patients suffering from age-related macular degeneration, has inspired further clinical trials on hPSCs (Schwartz et al., 2015; Song et al., 2015), including one in Japan using autologous iPSCs (Mandai et al., 2017). However, the high tumorigenic potential of undifferentiated hPSCs, due to unlimited proliferation and pluripotency leading to teratoma in vivo, is an important technical hurdle preventing safe stem cell therapy (Blum and Benvenisty, 2008; Ben-David and Benvenisty, 2011). Thus, a variety of techniques using small molecules (Ben-David et al., 2013; Lee et al., 2013), antibodies (Choo et al., 2008), and genetic approaches (Cho et al., 2015; Yagyu et al., 2015) have been developed 
to selectively eliminate or sequester undesirable residual hPSCs prior to engraftment in patients (Jeong et al., 2017a). For example, YM155 (1-(2-methoxyethyl)-2-methyl-4,9-dioxo-3(pyrazin-2-ylmethyl)-4,9- dihydro-1H-naphtho[2,3-d]imidazol3 -ium bromide) can effectively eliminate undifferentiated hPSCs by inducing p53-dependent mitochondria cell death without affecting the functionality of differentiated cells (Lee et al., 2013). The selective cytotoxic efficacy toward undifferentiated hPSCs and safety regarding differentiated cells, termed stemotoxic activity, has been confirmed by independent studies (Bedel et al., 2017; Kang et al., 2017; Kim K. T. et al., 2017). YM155 was originally developed as an anti-cancer drug that targets Survivin (encoded by BIRC5) (Nakahara et al., 2007), which is highly expressed in many cancer cell types, as well as hPSCs (Lee et al., 2013). The high selectivity of YM155 toward hPSCs cannot be completely explained by suppression of BIRC5 because p53 accumulation and consequent cell death occurs prior to dramatic suppression of BIRC5 expression (Lee et al., 2013). Recent studies reported that YM155 is imported through solute carrier family 35 member F2 (SLC35F2) in cancer cells prior to induction of DNA damage, and SLC35F2 expression determines the cytotoxicity of YM155 against cancer cells (Winter et al., 2014). Persistent DNA damage by YM155 (Wani et al., 2018b) results from redox-activated oxidative DNA damage (Wani et al., 2018a) or inhibition of topoisomerase (Hong et al., 2017), independent of the Survivin expression level (Sim et al., 2017). Analysis of the cytotoxicity of YM155 analogs in lung cancer cell lines, involving structure-activity relationship (SAR) studies on YM155, revealed that the quinone moiety and the positively charged imidazolium ring in the tricyclic naphthoimidazolium scaffold is important for cytotoxicity (Ho et al., 2015). The same analogs were also tested against two human embryonic carcinoma cell lines and compared with IMR-90 lung fibroblast cells (Ho et al., 2016). In the present study, we synthesized 26 analogs of YM155, in which the pyrazinylmethyl group was substituted with alkyl, hydroxyalkyl, aminoalkyl, substituted phenyl, and substituted benzyl groups, and we tested their stemotoxic activity toward hPSCs compared with isogenic smooth muscle cells (SMCs). We found that nitrogen in the pyrazine ring structure of YM155 serves as a hydrogen bond acceptor, and the interactions are critical for the stemotoxic activity of YM155 via uptake by SLC35F2.

\section{MATERIALS AND METHODS}

\section{Chemistry \\ General Information}

Unless stated otherwise, all reactions were performed under argon atmosphere with dry solvents under anhydrous conditions. Tetrahydrofuran and $\mathrm{Et}_{2} \mathrm{O}$ were distilled immediately before use of sodium benzophenone ketyl. Dichloromethane, chloroform, triethylamine, acetonitrile, and pyridine were freshly distilled from calcium hydride. All starting materials and reagents were obtained from commercial suppliers and were used without further purification, unless otherwise noted. Solvents for routine isolation of products and chromatography were reagent grade and glass distilled. Silica gel 60 (230-400 mesh,
Merck) was used for flash column chromatography. The reaction progress was monitored by thin-layer chromatography (TLC), which was performed using $0.25 \mathrm{~mm}$ silica gel plates (Merck). Optical rotations were measured with a JASCO P-2000 digital polarimeter at ambient temperature using $100 \mathrm{~mm}$ cell of $2 \mathrm{~mL}$ capacity. ${ }^{1} \mathrm{H}$ and ${ }^{13} \mathrm{C}$ NMR spectra were recorded on JEOL JNM-LA 300, BRUKER AVANCE-500, BRUKER AVANCE-400, JEOL JNM-ECA-600, and BRUKER AVANCE-800. ${ }^{1} \mathrm{H}-\mathrm{NMR}$ data were reported as follows: chemical shift (parts per million, $\delta$ ), multiplicity (br, broad signal; s, singlet; d, doublet; t, triplet; q, quartet; quint, quintet; $\mathrm{m}$, multiplet and/or multiple resonances), coupling constant in hertz $(\mathrm{Hz})$, and number of protons. Infrared spectra were recorded on a JASCO FT-IR-4200 spectrometer and are reported in frequency of absorption $\left(\mathrm{cm}^{-1}\right)$. High resolution mass spectra were obtained with JEOL JMS-700 instrument and Agilent Q TOF 6530.

\section{Representative Synthetic Procedure of YM Analogs 2-Chloro-3-((2-methoxyethyl)amino)naphthalene-1,4- dione (2)}

Methoxyethylamine (2 equiv.) was added to a stirred solution of 1 and triethylamine ( 2 equiv.) in DCM and then stirred another $5 \mathrm{~h}$. Water was added to the reaction mixture and the organic layer was separated, washed with water (2 times), and dried over $\mathrm{MgSO}_{4}$. Solvent was removed under reduced pressure and purified by silica gel column chromatography (ethyl acetate: hexanes $=1: 4)$ to afford 2 as red solid.

${ }^{1} \mathrm{H}$ NMR $\left(600 \mathrm{MHz}, \mathrm{CDCl}_{3}\right) \delta 8.02(\mathrm{dd}, J=7.8,0.9 \mathrm{~Hz}, 1 \mathrm{H})$, $7.91(\mathrm{~d}, J=7.4 \mathrm{~Hz}, 1 \mathrm{H}), 7.62(\mathrm{td}, J=7.6,1.4 \mathrm{~Hz}, 1 \mathrm{H}), 7.53(\mathrm{td}$, $J=7.6,1.4 \mathrm{~Hz}, 1 \mathrm{H}), 6.29$ (bs, $1 \mathrm{H}), 3.97(\mathrm{t}, J=5.3 \mathrm{~Hz}, 2 \mathrm{H}), 3.56$ $(\mathrm{t}, J=5.4 \mathrm{~Hz}, 2 \mathrm{H}), 3.35(\mathrm{~s}, 3 \mathrm{H}) ;{ }^{13} \mathrm{C} \mathrm{NMR}\left(150 \mathrm{MHz}, \mathrm{CDCl}_{3}\right) \delta$ $180.1,180.0,176.5,144.1,134.7,132.4,132.3,129.6,126.6,126,5$, $71.1,71.0,58.8,44.3,44.2$.

\section{$N$-(3-Chloro-1,4-dioxo-1,4-dihydronaphthalen-2-yl)-N-(2- methoxyethyl)acetamide (3)}

Five drops of $c-\mathrm{H}_{2} \mathrm{SO}_{4}$ were added to a stirred solution of 2 in acetic anhydride $(10 \mathrm{~mL})$ and then stirred for $4 \mathrm{hrs}$. Acetic anhydride was removed under reduced pressure and purified by silica gel column chromatography (ethyl acetate: hexanes $=1: 2$ to $1: 1)$ to afford 3 as a yellowish solid.

${ }^{1} \mathrm{H}$ NMR $\left(600 \mathrm{MHz}, \mathrm{CD}_{3} \mathrm{OD}\right) \delta 8.18-8.16(\mathrm{~m}, 2 \mathrm{H}), 7.89-$ $7.86(\mathrm{~m}, 2 \mathrm{H}), 7.64-7.51(\mathrm{~m}, 2 \mathrm{H}), 4.12(\mathrm{dt}, J=14.6,3.7 \mathrm{~Hz}$, $1 \mathrm{H}), 3.81-3.94(\mathrm{~m}, 1 \mathrm{H}), 3.58-3.41(\mathrm{~m}, 2 \mathrm{H}), 3.00(\mathrm{~s}, 3 \mathrm{H}), 1.93$ (s, 3H); ${ }^{13} \mathrm{C}$ NMR (150 MHz, CD $\left.3 \mathrm{OD}\right) \delta 181.5,179.8,173.1$, $147.6,143.7,136.6,136.5,136.2,133.7,133.2,129.0,128.9,72.7$, $59.4,48.7,23.0$.

\section{N-(3-(Benzylamino)-1,4-dioxo-1,4-dihydronaphthalen-2- yl)-N-(2-methoxyethyl)acetamide (4c)}

Triethylamine ( 2 equiv.) and benzylamine ( 2 equiv.) were added to a stirred solution of 3 in DCM and then stirred for $10 \mathrm{~h}$. Water was added to the reaction mixture and the organic layer was separated, washed with water (2 times), and dried over $\mathrm{MgSO}_{4}$. Solvent was removed under reduced pressure and the crude material was purified by silica gel column chromatography 
(ethyl acetate: hexanes $=1: 1$ to ethyl acetate only) to afford $4 \mathrm{c}$ as a red oil.

${ }^{1} \mathrm{H}$ NMR $\left(500 \mathrm{MHz}, \mathrm{DMSO}-\mathrm{d}_{6}\right) \delta 7.98(\mathrm{~d}, J=8.0 \mathrm{~Hz}, 1 \mathrm{H})$, $7.93-7.89(\mathrm{~m}, 2 \mathrm{H}), 7.79(\mathrm{td}, J=7.7,1.2 \mathrm{~Hz}, 1 \mathrm{H}), 7.71(\mathrm{td}, J=7.5$, $1.2 \mathrm{~Hz}, 1 \mathrm{H}), 7.27$ (t, J=7.5 Hz, 1H), 7.20-7.18 (m, 3H), 4.67-4.56 (m, 2H), 3.76 (bs, $1 \mathrm{H}), 3.42-3.38(\mathrm{~m}, 1 \mathrm{H}), 3.31(\mathrm{~s}, 1 \mathrm{H}), 3.21-3.08$ (m, 2H), $3.00(\mathrm{~s}, 3 \mathrm{H}) ;{ }^{13} \mathrm{C}$ NMR (125 MHz, DMSO-d $\left.\mathrm{d}_{6}\right) \delta 182.8$, $170.0,172.0,144.0,140.0,135.5,133.2,132.5,131.0,128.9,127.5$, $127.1,127.0,126.3,117.7,69.4,58.3,47.6,47.0,21.5$.

\section{3-Benzyl-1-(2-methoxyethyl)-2-methyl-4,9-dioxo-4,9- dihydro-1H-naphtho[2,3-d]imidazol-3-ium bromide (5c)}

c- $\mathrm{HBr}$ (2 equiv.) was added to a stirred solution of $\mathbf{4 c}$ in $\mathrm{EtOH}$ and then refluxed for $12 \mathrm{~h}$. EtOH was removed under reduced pressure and ethyl acetate was added and stirred overnight. Precipitate formed was filtered and additional purification was performed by silica gel column chromatography (DCM: $\mathrm{MeOH}$ $=10: 1)$ to afford $5 \mathrm{c}$ as a yellow solid.

1-(2-Methoxyethyl)-2-methyl-4,9-dioxo-3-(4methoxyphenyl)-4,9-dihydro-1H-naphtho[2,3-d]imidazol-3ium bromide (5a)

${ }^{1} \mathrm{H} \mathrm{NMR}\left(300 \mathrm{MHz}, \mathrm{CDCl}_{3}\right) \delta 8.27(\mathrm{~m}, 1 \mathrm{H}), 8.03(\mathrm{~m}, 1 \mathrm{H}), 7.84-$ $7.72(\mathrm{~m}, 4 \mathrm{H}), 7.10-7.04(\mathrm{~m}, 2 \mathrm{H}), 4.94(\mathrm{t}, J=4.8 \mathrm{~Hz}, 2 \mathrm{H}), 3.90$ $(\mathrm{t}, J=4.8 \mathrm{~Hz}, 2 \mathrm{H}), 3.88(\mathrm{~s}, 3 \mathrm{H}), 3.33(\mathrm{~s}, 3 \mathrm{H}), 2.85(\mathrm{~s}, 3 \mathrm{H}) ;{ }^{13} \mathrm{C}$ NMR $\left(125 \mathrm{MHz}, \mathrm{CD}_{3} \mathrm{OD}\right) \delta 174.9,174.5,157.4,154.2,134.9$, $131.9,130.7,130.4,129.4,126.8,120.8,120.5,110.8,69.9,58.0$, $54.7,47.9,47.1,10.2$.

3-(3,4-dimethoxyphenyl)-1-(2-methoxyethyl)-2-methyl-4,9dioxo-4,9-dihydro-1H-naphtho[2,3-d]imidazol-3-ium bromide (5b)

${ }^{1} \mathrm{H}$ NMR (500 MHz, CD 3 OD) $\delta 8.26-8.10$ (m, 2H), 7.89 (bs, 2H), 7.32 (bs, $1 \mathrm{H}), 7.18$ (bs, 2H), 4.94 (bs, 2H), 3.93-3.84 (m, 8H), 3.88 (s, 3H), $3.36(\mathrm{~s}, 3 \mathrm{H}), 2.68(\mathrm{~s}, 3 \mathrm{H}) ;{ }^{13} \mathrm{C} \mathrm{NMR}\left(125 \mathrm{MHz}, \mathrm{CD}_{3} \mathrm{OD}\right) \delta$ 175.1, 173.1, 154.3, 151.5, 150.0, 134.9, 134.7, 132.0, 131.5, 126.8, $126.7,125.3,119.3,111.4,110.3,69.9,58.1,55.6,55.4,48.3,10.3$.

\section{3-Benzyl-1-(2-methoxyethyl)-2-methyl-4,9-dioxo-4,9- dihydro-1H-naphtho[2,3-d]imidazol-3-ium bromide (5c)}

${ }^{1} \mathrm{H}$ NMR (600 MHz, CD 3 OD) $\delta 8.27-8.24(\mathrm{~m}, 2 \mathrm{H}), 7.96-7.92(\mathrm{~m}$, $2 \mathrm{H}), 7.42-7.34(\mathrm{~m}, 5 \mathrm{H}), 6.06(\mathrm{~s}, 2 \mathrm{H}), 4.93(\mathrm{t}, J=4.8 \mathrm{~Hz}, 2 \mathrm{H})$, $3.86(\mathrm{t}, J=4.8 \mathrm{~Hz}, 2 \mathrm{H}), 3.30(\mathrm{~s}, 3 \mathrm{H}), 2.82(\mathrm{~m}, 3 \mathrm{H}) ;{ }^{13} \mathrm{C} \mathrm{NMR}$ $\left(150 \mathrm{MHz}, \mathrm{CD}_{3} \mathrm{OD}\right) \delta 176.3,176.2,155.2,136.3,136.2,134.5$, $133.4,133.4,131.9,131.7,130.4,129.9,128.3,128.3,128.2,71.1$, 59.4, 51.5, 49.4, 10.8; HR-MS (ESI+) calcd for $\mathrm{C}_{22} \mathrm{H}_{21} \mathrm{~N}_{2} \mathrm{O}_{3}[\mathrm{M}-$ $\left.\mathrm{Br}^{-}\right]^{+}$361.1547, found 361.1527.

3-(2-Fluorobenzyl)-1-(2-methoxyethyl)-2-methyl-4,9-dioxo4,9-dihydro-1H-naphtho[2,3-d]imidazol-3-ium bromide (5d)

${ }^{1} \mathrm{H}$ NMR $\left(600 \mathrm{MHz}, \mathrm{CDCl}_{3}\right) \delta 8.27-8.25(\mathrm{~m}, 1 \mathrm{H}), 8.23-8.22(\mathrm{~m}$, $1 \mathrm{H}), 7.96-7.91(\mathrm{~m}, 2 \mathrm{H}), 7.43(\mathrm{td}, J=7.8,7.3 \mathrm{~Hz}, 1 \mathrm{H}), 7.36(\mathrm{t}$, $J=7.8 \mathrm{~Hz}, 1 \mathrm{H}), 7.23-7.19(\mathrm{~m}, 2 \mathrm{H}), 6.10(\mathrm{~s}, 2 \mathrm{H}), 4.95(\mathrm{t}, J=$ $5.0 \mathrm{~Hz}, 2 \mathrm{H}), 3.87(\mathrm{t}, J=5.0 \mathrm{~Hz}, 2 \mathrm{H}), 3.32(\mathrm{~s}, 3 \mathrm{H}), 2.90(\mathrm{~s}, 3 \mathrm{H})$;
${ }^{13} \mathrm{C}$ NMR $\left(150 \mathrm{MHz}, \mathrm{CD}_{3} \mathrm{OD}\right) \delta 176.2,176.0,161.9\left(\mathrm{~d}, J_{\mathrm{C}-\mathrm{F}}=\right.$ $244.9 \mathrm{~Hz}), 155.5,136.3,136.3,133.3\left(\mathrm{~d}, J_{\mathrm{C}-\mathrm{F}}=15.8 \mathrm{~Hz}\right), 132.3$, $132.3,132.0,131.7,130.6\left(\mathrm{~d}, J_{\mathrm{C}-\mathrm{F}}=2.9 \mathrm{~Hz}\right), 128.3,128.3,126.1$ $\left(\mathrm{d}, J_{\mathrm{C}-\mathrm{F}}=3.6 \mathrm{~Hz}\right), 121.5\left(\mathrm{~d}, J_{\mathrm{C}-\mathrm{F}}=13.7 \mathrm{~Hz}\right), 117.0\left(\mathrm{~d}, J_{\mathrm{C}-\mathrm{F}}\right.$ $=20.8 \mathrm{~Hz}$ ), 71.1, 59.4, 49.6, 46.6, 11.3; HR-MS (ESI+) calcd for $\mathrm{C}_{22} \mathrm{H}_{20} \mathrm{FN}_{2} \mathrm{O}_{3}\left[\mathrm{M}-\mathrm{Br}^{-}\right]^{+}$379.1452, found 379.1440 .

3-(3-Fluorobenzyl)-1-(2-methoxyethyl)-2-methyl-4,9-dioxo4,9-dihydro-1H-naphtho[2,3-d]imidazol-3-ium bromide (5e)

${ }^{1} \mathrm{H}$ NMR (300 MHz, CD $\left.{ }_{3} \mathrm{OD}\right) \delta 8.38-8.23(\mathrm{~m}, 2 \mathrm{H}), 8.02-7.86$ (m, 2H), 7.54-7.32 (m, 1H), 7.32-6.98 (m, 3H), $6.07(\mathrm{~s}, 2 \mathrm{H}), 4.94$ $(\mathrm{t}, J=4.9 \mathrm{~Hz}, 2 \mathrm{H}), 3.87(\mathrm{t}, J=4.9 \mathrm{~Hz}, 2 \mathrm{H}), 3.32(\mathrm{~s}, 3 \mathrm{H}), 2.85$ $(\mathrm{m}, 3 \mathrm{H}) ;{ }^{13} \mathrm{C} \mathrm{NMR}\left(75 \mathrm{MHz}, \mathrm{CD}_{3} \mathrm{OD}\right) \delta 176.3,176.1,164.5(\mathrm{~d}$, $\left.J_{\mathrm{C}-\mathrm{F}}=246.3 \mathrm{~Hz}\right), 155.2,137.0\left(\mathrm{~d}, J_{\mathrm{C}-\mathrm{F}}=7.6 \mathrm{~Hz}\right), 136.3,136.2$, $133.3,133.3,132.3\left(\mathrm{~d}, J_{\mathrm{C}-\mathrm{F}}=8.4 \mathrm{~Hz}\right), 131.9,131.6,128.2,124.1$ $\left(\mathrm{d}, J_{\mathrm{C}-\mathrm{F}}=3.0 \mathrm{~Hz}\right), 116.7\left(\mathrm{~d}, J_{\mathrm{C}-\mathrm{F}}=21.2 \mathrm{~Hz}\right), 115.2\left(\mathrm{~d}, J_{\mathrm{C}-\mathrm{F}}\right.$ $=23.2 \mathrm{~Hz}$ ), 71.1, 59.4, 50.9, 49.4, 11.4; HR-MS (ESI+) calcd for $\mathrm{C}_{22} \mathrm{H}_{20} \mathrm{FN}_{2} \mathrm{O}_{3}\left[\mathrm{M}-\mathrm{Br}^{-}\right]^{+} 379.1452$, found 379.1470 .

3-(4-Fluorobenzyl)-1-(2-methoxyethyl)-2-methyl-4,9-dioxo4,9-dihydro-1H-naphtho[2,3-d]imidazol-3-ium bromide (5f)

${ }^{1} \mathrm{H}$ NMR $(300 \mathrm{MHz}$, DMSO-d 6 ) $\delta 8.22-8.16(\mathrm{~m}, 2 \mathrm{H}), 8.04-7.99$ $(\mathrm{m}, 2 \mathrm{H}), 7.42(\mathrm{dd}, J=8.9,5.6 \mathrm{~Hz}, 2 \mathrm{H}), 7.25(\mathrm{t}, J=9.0 \mathrm{~Hz}$, $2 \mathrm{H}), 6.02(\mathrm{~s}, 2 \mathrm{H}), 4.86(\mathrm{t}, J=4.8 \mathrm{~Hz}, 2 \mathrm{H}), 3.79(\mathrm{t}, J=4.8 \mathrm{~Hz}$, $2 \mathrm{H}), 3.23(\mathrm{~s}, 3 \mathrm{H}), 2.86(\mathrm{~s}, 3 \mathrm{H}) ;{ }^{13} \mathrm{C}$ NMR (75 MHz, DMSO-d 6 ) $\delta 174.9,174.8,162.0\left(\mathrm{~d}, J_{\mathrm{C}-\mathrm{F}}=243.3 \mathrm{~Hz}\right), 153.3,135.3,135.3$, $131.7,131.7,130.5,130.0,129.8\left(\mathrm{~d}, J_{\mathrm{C}-\mathrm{F}}=3.2 \mathrm{~Hz}\right), 129.4(\mathrm{~d}$, $\left.J_{\mathrm{C}-\mathrm{F}}=8.3 \mathrm{~Hz}\right), 126.9,115.8\left(\mathrm{~d}, J_{\mathrm{C}-\mathrm{F}}=21.7 \mathrm{~Hz}\right), 69.5,58.5,48.9$, 47.5, 10.7; HR-MS (ESI+) calcd for $\mathrm{C}_{22} \mathrm{H}_{20} \mathrm{FN}_{2} \mathrm{O}_{3}\left[\mathrm{M}-\mathrm{Br}^{-}\right]^{+}$ 379.1452 , found 379.1469 .

3-(4-Chlorobenzyl)-1-(2-methoxyethyl)-2-methyl-4,9-dioxo4,9-dihydro-1H-naphtho[2,3-d]imidazol-3-ium bromide (5g)

${ }^{1} \mathrm{H}$ NMR (300 MHz, CD $\left.{ }_{3} \mathrm{OD}\right) \delta 8.30-8.21(\mathrm{~m}, 2 \mathrm{H}), 7.98-7.90(\mathrm{~m}$, $2 \mathrm{H}), 7.43-7.36(\mathrm{~m}, 4 \mathrm{H}), 6.04(\mathrm{~s}, 2 \mathrm{H}), 4.93(\mathrm{t}, J=4.9 \mathrm{~Hz}, 2 \mathrm{H}), 3.86$ $(\mathrm{t}, J=4.8 \mathrm{~Hz}, 2 \mathrm{H}), 3.31(\mathrm{~s}, 3 \mathrm{H}), 2.86(\mathrm{~m}, 3 \mathrm{H}) ;{ }^{13} \mathrm{C} \mathrm{NMR}(75 \mathrm{MHz}$, $\left.\mathrm{CD}_{3} \mathrm{OD}\right) \delta 176.3,176.2,155.2,136.3,136.3,135.8,133.4,133.3$, 133.2, 132.0, 131.6, 130.4, 130.1, 128.3, 71.1, 59.4, 50.9, 49.4, 11.1; HR-MS (ESI+) calcd for $\mathrm{C}_{22} \mathrm{H}_{20} \mathrm{ClN}_{2} \mathrm{O}_{3}\left[\mathrm{M}-\mathrm{Br}^{-}\right]^{+} 395.1157$, found 395.1182 .

\section{3-(2-Methoxybenzyl)-1-(2-methoxyethyl)-2-methyl-4,9-} dioxo-4,9-dihydro-1H-naphtho[2,3-d]imidazol-3-ium bromide (5h)

${ }^{1} \mathrm{H}$ NMR (400 MHz, CD $\left.3 \mathrm{OD}\right) \delta 8.27-8.21$ (m, 2H), 7.95-7.91 (m, $2 \mathrm{H}), 7.37(\mathrm{td}, J=7.9,1.5 \mathrm{~Hz}, 1 \mathrm{H}), 7.30(\mathrm{~d}, J=7.3 \mathrm{~Hz}, 1 \mathrm{H}), 7.04$ $(\mathrm{d}, J=8.2 \mathrm{~Hz}, 1 \mathrm{H}), 6.97(\mathrm{t}, J=7.5 \mathrm{~Hz}, 1 \mathrm{H}), 5.98(\mathrm{~s}, 2 \mathrm{H}), 4.92(\mathrm{t}, J$ $=4.8 \mathrm{~Hz}, 2 \mathrm{H}), 3.86(\mathrm{t}, J=4.8 \mathrm{~Hz}, 2 \mathrm{H}), 3.81(\mathrm{~s}, 3 \mathrm{H}), 3.31(\mathrm{~s}, 3 \mathrm{H})$, $2.84(\mathrm{~m}, 3 \mathrm{H}) ;{ }^{13} \mathrm{C} \mathrm{NMR}\left(150 \mathrm{MHz}, \mathrm{CD}_{3} \mathrm{OD}\right) \delta 176.2,175.9,158.8$, $155.5,136.2,133.3,132.1,131.8,131.8,130.8,128.2,128.2,122.1$, $121.9,112.2,71.3,59.4,56.1,49.5,48.4,10.9$; HR-MS (ESI+) calcd for $\mathrm{C}_{23} \mathrm{H}_{23} \mathrm{~N}_{2} \mathrm{O}_{4}\left[\mathrm{M}-\mathrm{Br}^{-}\right]^{+}$391.1652, found 391.1631. 
3-(3-Methoxybenzyl)-1-(2-methoxyethyl)-2-methyl-4,9dioxo-4,9-dihydro-1H-naphtho[2,3-d]imidazol-3-ium bromide (5i)

${ }^{1} \mathrm{H}$ NMR (400 MHz, CD 3 OD) $\delta 8.26(\mathrm{td}, J=7.5,4.1 \mathrm{~Hz}, 2 \mathrm{H})$, 8.01-7.87 (m, 2H), $7.31(\mathrm{td}, J=7.6,1.1 \mathrm{~Hz}, 1 \mathrm{H}), 6.95-6.89(\mathrm{~m}$, $1 \mathrm{H}), 6.91(\mathrm{~s}, 1 \mathrm{H}), 6.86(\mathrm{~d}, J=7.7 \mathrm{~Hz}, 1 \mathrm{H}), 6.03(\mathrm{~s}, 2 \mathrm{H}), 4.93$ $(\mathrm{t}, J=4.8 \mathrm{~Hz}, 2 \mathrm{H}), 3.86(\mathrm{t}, J=4.9 \mathrm{~Hz}, 2 \mathrm{H}), 3.78(\mathrm{~s}, 3 \mathrm{H}), 3.31$ $(\mathrm{s}, 3 \mathrm{H}), 2.83(\mathrm{~s}, 3 \mathrm{H}) ;{ }^{13} \mathrm{C} \mathrm{NMR}\left(100 \mathrm{MHz}, \mathrm{CD}_{3} \mathrm{OD}\right) \delta 176.3$, $176.2,161.8,155.2,136.3,136.2,135.9,133.4,131.9,131.6,131.5$, 128.3, 128.2, 120.1, 115.1, 114.2, 71.1, 59.4, 55.9, 51.3, 49.4, 10.9; HR-MS (ESI+) calcd for $\mathrm{C}_{23} \mathrm{H}_{23} \mathrm{~N}_{2} \mathrm{O}_{4}\left[\mathrm{M}-\mathrm{Br}^{-}\right]^{+}$391.1652, found 391.1629.

3-(4-Methoxybenzyl)-1-(2-methoxyethyl)-2-methyl-4,9dioxo-4,9-dihydro-1H-naphtho[2,3-d]imidazol-3-ium bromide (5j)

${ }^{1} \mathrm{H}$ NMR $\left(800 \mathrm{MHz}, \mathrm{CDCl}_{3}\right) \delta 8.19-8.14(\mathrm{~m}, 2 \mathrm{H}), 7.82(\mathrm{dd}, J=$ 5.7, 3.3 Hz, 2H), $7.25(\mathrm{~d}, J=8.8 \mathrm{~Hz}, 2 \mathrm{H}), 6.85(\mathrm{~d}, J=8.8 \mathrm{~Hz}, 2 \mathrm{H})$, $6.00(\mathrm{~s}, 2 \mathrm{H}), 4.93(\mathrm{t}, J=4.9 \mathrm{~Hz}, 2 \mathrm{H}), 3.78(\mathrm{t}, J=5.0 \mathrm{~Hz}, 2 \mathrm{H}), 3.73$ (s, 3H), 3.23 (s, 3H), 3.03 (s, 3H); $\left.{ }^{13} \mathrm{C} \mathrm{NMR} \mathrm{(200} \mathrm{MHz,} \mathrm{CDCl}_{3}\right) \delta$ $174.7,174.7,160.0,155.0,152.0,135.2,135.2,131.6,130.2,130.1$, 129.0, 127.6, 127.4, 124.4, 114.6, 69.8, 59.1, 55.3, 50.9, 48.8, 13.2; HR-MS (ESI+) calcd for $\mathrm{C}_{23} \mathrm{H}_{23} \mathrm{~N}_{2} \mathrm{O}_{4}\left[\mathrm{M}-\mathrm{Br}^{-}\right]^{+} 391.1652$, found 391.1631 .

1-(2-Methoxyethyl)-2-methyl-4,9-dioxo-3-(pyridin-2ylmethyl)-4,9-dihydro-1H-naphtho[2,3-d]imidazol-3-ium bromide (5k)

${ }^{1} \mathrm{H}$ NMR (800 MHz, DMSO-d 6 ) $\delta 8.53(\mathrm{~d}, J=4.6 \mathrm{~Hz}, 1 \mathrm{H}), 8.17$ $(\mathrm{dd}, J=7.6,0.9 \mathrm{~Hz}, 1 \mathrm{H}), 8.09$ (dd, $J=7.5,1.0 \mathrm{~Hz}, 1 \mathrm{H}), 8.01-$ $7.94(\mathrm{~m}, 3 \mathrm{H}), 7.70(\mathrm{~d}, J=8.0 \mathrm{~Hz}, 1 \mathrm{H}), 7.46(\mathrm{dd}, J=7.1,5.3 \mathrm{~Hz}$, $1 \mathrm{H}), 6.15(\mathrm{~s}, 2 \mathrm{H}), 4.92(\mathrm{t}, J=4.9 \mathrm{~Hz}, 2 \mathrm{H}), 3.82(\mathrm{t}, J=4.9 \mathrm{~Hz}$, 2H), 3.26 (s, 3H), 2.97 (s, 3H); ${ }^{13} \mathrm{C}$ NMR (200 MHz, CD $\left.3 \mathrm{OD}\right) \delta$ $174.7,174.6,154.2,152.0,148.6,138.7,135.4,135.2,131.8,131.4$, 130.3, 130.0, 127.0, 126.8, 124.0, 122.8, 69.6, 58.6, 48.6, 47.7, 11.0; HR-MS (ESI+) calcd for $\mathrm{C}_{21} \mathrm{H}_{20} \mathrm{~N}_{3} \mathrm{O}_{3}\left[\mathrm{M}-\mathrm{Br}^{-}\right]^{+}$362.1499, found 362.1476 .

1-(2-Methoxyethyl)-2-methyl-4,9-dioxo-3-(pyridin-3ylmethyl)-4,9-dihydro-1H-naphtho[2,3-d]imidazol-3-ium bromide (5l)

${ }^{1} \mathrm{H}$ NMR $\left(600 \mathrm{MHz}, \mathrm{CD}_{3} \mathrm{OD}\right) \delta 8.65(\mathrm{~d}, J=2.2 \mathrm{~Hz}, 1 \mathrm{H}), 8.56(\mathrm{dd}$, $J=4.9,1.4 \mathrm{~Hz}, 1 \mathrm{H}), 8.29-8.23(\mathrm{~m}, 2 \mathrm{H}), 7.97-7.91(\mathrm{~m}, 2 \mathrm{H}), 7.87$ $(\mathrm{dd}, J=6.5,1.7 \mathrm{~Hz}, 1 \mathrm{H}), 7.48(\mathrm{dd}, J=8.1,4.9 \mathrm{~Hz}, 1 \mathrm{H}), 6.09(\mathrm{~s}$, $2 \mathrm{H}), 4.94(\mathrm{t}, J=4.9 \mathrm{~Hz}, 2 \mathrm{H}), 3.87(\mathrm{t}, J=4.9 \mathrm{~Hz}, 2 \mathrm{H}), 3.32(\mathrm{~s}, 3 \mathrm{H})$, $2.91(\mathrm{~s}, 3 \mathrm{H}) ;{ }^{13} \mathrm{C}$ NMR $\left(150 \mathrm{MHz}, \mathrm{CD}_{3} \mathrm{OD}\right) \delta 176.3,176.2,155.3$, $150.6,149.4,137.4,136.4,136.3,133.4,133.3,132.0,131.7,131.3$, 128.3, 128.3, 125.7, 71.1, 59.4, 49.5, 49.3, 10.8; HR-MS (ESI+) calcd for $\mathrm{C}_{21} \mathrm{H}_{20} \mathrm{~N}_{3} \mathrm{O}_{3}\left[\mathrm{M}-\mathrm{Br}^{-}\right]^{+}$362.1499, found 362.1488 .

1-(2-Methoxyethyl)-2-methyl-4,9-dioxo-3-(pyridin-4ylmethyl)-4,9-dihydro-1H-naphtho[2,3-d]imidazol-3-ium bromide (5m)

${ }^{1} \mathrm{H}$ NMR (800 MHz, DMSO-d 6 ) $\delta 8.76$ (br s, 2H), 8.22 (dd, $J=7.6,1.2 \mathrm{~Hz}, 1 \mathrm{H}), 8.11(\mathrm{dd}, J=7.5,1.3 \mathrm{~Hz}, 1 \mathrm{H}), 8.02(\mathrm{td}$, $J=7.5,1.4 \mathrm{~Hz}, 1 \mathrm{H}), 7.99(\mathrm{td}, J=7.5,1.4 \mathrm{~Hz}, 1 \mathrm{H}), 7.58(\mathrm{~s}$, $2 \mathrm{H}), 6.15(\mathrm{~s}, 2 \mathrm{H}), 4.89(\mathrm{t}, J=5.0 \mathrm{~Hz}, 2 \mathrm{H}), 3.81(\mathrm{t}, J=5.0 \mathrm{~Hz}$,
2H), $3.26(\mathrm{~s}, 3 \mathrm{H}), 2.86(\mathrm{~s}, 3 \mathrm{H}) ;{ }^{13} \mathrm{C}$ NMR (200 MHz, DMSO$\left.\mathrm{d}_{6}\right) \delta 174.8,174.7,153.9,147.0,135.4,135.3,131.8,131.5,130.5$, $130.1,127.0,126.8,122.7,122.6,69.5,58.5,49.0,47.7,10.5$; HR-MS (ESI+) calcd for $\mathrm{C}_{21} \mathrm{H}_{20} \mathrm{~N}_{3} \mathrm{O}_{3}\left[\mathrm{M}-\mathrm{Br}^{-}\right]^{+}$362.1499, found 362.1532 .

\section{3-(4-Hydroxyphenethyl)-1-(2-methoxyethyl)-2-methyl-4,9-} dioxo-4,9-dihydro-1H-naphtho[2,3-d]imidazol-3-ium bromide (5n)

${ }^{1} \mathrm{H}$ NMR (800 MHz, DMSO-d 6 ) $\delta 9.36$ (s, 1H), 8.21 (br s, $\left.2 \mathrm{H}\right)$, 8.02 (br s, $2 \mathrm{H}), 7.01(\mathrm{~d}, J=8.4 \mathrm{~Hz}, 2 \mathrm{H}), 6.69(\mathrm{~d}, J=8.4 \mathrm{~Hz}$, $2 \mathrm{H}), 4.79$ (br s, $2 \mathrm{H}), 3.72(\mathrm{t}, J=4.9 \mathrm{~Hz}, 2 \mathrm{H}), 3.34(\mathrm{~s}, 3 \mathrm{H}), 3.24$ $(\mathrm{s}, 3 \mathrm{H}), 3.02(\mathrm{t}, J=6.7 \mathrm{~Hz}, 2 \mathrm{H}), 2.41(\mathrm{br} \mathrm{s}, 2 \mathrm{H}) ;{ }^{13} \mathrm{C}$ NMR $(200$ MHz, DMSO-d $\left.\mathrm{d}_{6}\right) \delta 174.8,174.6,156.5,152.5,135.2,135.2,131.8$, 131.6, 130.3, 130.0, 126.9, 126.5, 115.4, 69.6, 58.5, 49.2, 47.3, 33.7, 9.8; HR-MS (ESI+) calcd for $\mathrm{C}_{23} \mathrm{H}_{23} \mathrm{~N}_{2} \mathrm{O}_{4}\left[\mathrm{M}-\mathrm{Br}^{-}\right]^{+} 391.1652$, found 391.1637 .

1-(2-Methoxyethyl)-3-(4-methoxyphenethyl)-2-methyl-4,9dioxo-4,9-dihydro-1H-naphtho[2,3-d]imidazol-3-ium bromide (5o)

${ }^{1} \mathrm{H}$ NMR $\left(800 \mathrm{MHz}, \mathrm{CD}_{3} \mathrm{OD}\right) \delta 8.30-8.23(\mathrm{~m}, 2 \mathrm{H}), 8.01-7.86$ $(\mathrm{m}, 2 \mathrm{H}), 7.12(\mathrm{dd}, J=8.4,3.5 \mathrm{~Hz}, 2 \mathrm{H}), 6.84(\mathrm{~d}, J=8.5 \mathrm{~Hz}$, $2 \mathrm{H}), 4.88(\mathrm{t}, J=6.8 \mathrm{~Hz}, 2 \mathrm{H}), 4.84(\mathrm{t}, J=5.3 \mathrm{~Hz}, 2 \mathrm{H}), 3.80(\mathrm{t}$, $J=4.7 \mathrm{~Hz}, 2 \mathrm{H}), 3.73(\mathrm{~s}, 3 \mathrm{H}), 3.34(\mathrm{~s}, 3 \mathrm{H}), 3.31(\mathrm{~s}, 3 \mathrm{H}), 3.16$ $(\mathrm{t}, J=6.8 \mathrm{~Hz}, 2 \mathrm{H}) ;{ }^{13} \mathrm{C} \mathrm{NMR}\left(200 \mathrm{MHz}, \mathrm{CD}_{3} \mathrm{OD}\right) \delta 176.2$, 176.0, 160.6, 154.5, 136.2, 136.2, 133.4, 133.3, 131.7, 131.7, 131.5, $129.5,128.3,128.2,115.5,71.2,59.4,55.7,50.8,49.8,35.5,10.2$; HR-MS (ESI+) calcd for $\mathrm{C}_{24} \mathrm{H}_{25} \mathrm{~N}_{2} \mathrm{O}_{4}\left[\mathrm{M}-\mathrm{Br}^{-}\right]^{+} 405.1809$, found 405.1825 .

3-(Furan-2-ylmethyl)-1-(2-methoxyethyl)-2-methyl-4,9dioxo-4,9-dihydro-1H-naphtho[2,3-d]imidazol-3-ium bromide (5p)

${ }^{1} \mathrm{H}$ NMR (500 MHz, DMSO-d 6 ) $\delta 8.22$ (br s, $\left.1 \mathrm{H}\right), 8.18$ (br s, $\left.1 \mathrm{H}\right)$, $8.01($ br s, $2 \mathrm{H}), 7.72(\mathrm{~d}, J=1.0 \mathrm{~Hz}, 1 \mathrm{H}), 6.71(\mathrm{~d}, J=3.2 \mathrm{~Hz}, 1 \mathrm{H})$, $6.50(\mathrm{dd}, J=3.1,1.9 \mathrm{~Hz}, 1 \mathrm{H}), 6.04(\mathrm{~s}, 2 \mathrm{H}), 4.85(\mathrm{br} \mathrm{s}, 2 \mathrm{H}), 3.79(\mathrm{t}$, $J=4.9 \mathrm{~Hz}, 2 \mathrm{H}), 3.24(\mathrm{~s}, 3 \mathrm{H}), 3.01(\mathrm{~s}, 3 \mathrm{H}) ;{ }^{13} \mathrm{C} \mathrm{NMR}(125 \mathrm{MHz}$, DMSO-d 6 ) $\delta 174.6,174.5,153.1,146.4,144.3,135.3,135.2,131.7$, $131.4,130.4,129.4,126.9,126.8,111.0,110.9,69.4,58.4,47.5$, 43.1, 10.5; HR-MS (ESI+) calcd for $\mathrm{C}_{20} \mathrm{H}_{19} \mathrm{~N}_{2} \mathrm{O}_{4}\left[\mathrm{M}-\mathrm{Br}^{-}\right]^{+}$ 351.1339 , found 351.1326 .

1-(2-Methoxyethyl)-2-methyl-4,9-dioxo-3-(thiophen-2ylmethyl)-4,9-dihydro-1H-naphtho[2,3-d]imidazol-3-ium bromide (5q)

${ }^{1} \mathrm{H}$ NMR (400 MHz, CD $\left.3 \mathrm{OD}\right) \delta 8.32-8.30(\mathrm{~m}, 1 \mathrm{H}), 8.27-8.25$ $(\mathrm{m}, 1 \mathrm{H}), 7.97-7.94(\mathrm{~m}, 2 \mathrm{H}), 7.48(\mathrm{dd}, J=5.2,1.1 \mathrm{~Hz}, 1 \mathrm{H})$, $7.37(\mathrm{~d}, J=3.5 \mathrm{~Hz}, 1 \mathrm{H}), 7.04(\mathrm{dd}, J=5.1,3.6 \mathrm{~Hz}, 1 \mathrm{H}), 6.22$ $(\mathrm{s}, 2 \mathrm{H}), 4.90(\mathrm{t}, J=4.9 \mathrm{~Hz}, 2 \mathrm{H}), 3.84(\mathrm{t}, J=4.9 \mathrm{~Hz}, 2 \mathrm{H})$, $3.30(\mathrm{~s}, 3 \mathrm{H}), 3.02(\mathrm{~s}, 3 \mathrm{H}) ;{ }^{13} \mathrm{C} \mathrm{NMR}\left(100 \mathrm{MHz}, \mathrm{CD}_{3} \mathrm{OD}\right) \delta$ $176.4,176.1,154.8,136.4,136.3,136.0,133.4,133.3,131.9,131.0$, $130.5,128.9,128.3,128.3,128.3,71.1,59.3,49.4,46.6,11.5$; HR-MS (ESI+) calcd for $\mathrm{C}_{20} \mathrm{H}_{19} \mathrm{~N}_{2} \mathrm{O}_{3} \mathrm{~S}\left[\mathrm{M}-\mathrm{Br}^{-}\right]^{+} 367.1111$, found 367.1091. 
3-(2-Hydroxyethyl)-1-(2-methoxyethyl)-2-methyl-4,9-dioxo4,9-dihydro-1H-naphtho[2,3-d]imidazol-3-ium bromide (5r)

${ }^{1} \mathrm{H}$ NMR (800 MHz, CD 3 OD) $\delta 8.27-8.25$ (m, 2H), 7.95-7.93 (m, $2 \mathrm{H}), 4.92(\mathrm{t}, J=4.9 \mathrm{~Hz}, 2 \mathrm{H}), 4.83(\mathrm{t}, J=5.1 \mathrm{~Hz}, 2 \mathrm{H}), 4.00(\mathrm{t}, J=$ $5.0 \mathrm{~Hz}, 2 \mathrm{H}), 3.86(\mathrm{t}, J=5.0 \mathrm{~Hz}, 2 \mathrm{H}), 3.32(\mathrm{~s}, 3 \mathrm{H}), 2.92(\mathrm{~s}, 3 \mathrm{H})$; ${ }^{13} \mathrm{C}$ NMR $\left(200 \mathrm{MHz}, \mathrm{CD}_{3} \mathrm{OD}\right) \delta 176.3,176.2,155.6,136.2,133.3$, 133.3, 131.8, 131.8, 128.2, 128.2, 71.3, 60.9, 59.4, 51.4, 49.2, 11.2; HR-MS (ESI+) calcd for $\mathrm{C}_{17} \mathrm{H}_{19} \mathrm{~N}_{2} \mathrm{O}_{4}\left[\mathrm{M}-\mathrm{Br}^{-}\right]^{+}$315.1339, found 315.1326 .

3-(3-Hydroxypropyl)-1-(2-methoxyethyl)-2-methyl-4,9dioxo-4,9-dihydro-1H-naphtho[2,3-d]imidazol-3-ium bromide (5s)

${ }^{1} \mathrm{H}$ NMR (400 MHz, CD 3 OD) $\delta 8.27-8.22(\mathrm{~m}, 2 \mathrm{H}), 7.96-7.92(\mathrm{~m}$, $2 \mathrm{H}), 4.91(\mathrm{t}, J=4.9 \mathrm{~Hz}, 2 \mathrm{H}), 4.82(\mathrm{t}, J=7.0 \mathrm{~Hz}, 2 \mathrm{H}), 3.86(\mathrm{t}, J=$ $4.9 \mathrm{~Hz}, 2 \mathrm{H}), 3.70(\mathrm{t}, J=5.7 \mathrm{~Hz}, 2 \mathrm{H}), 3.33(\mathrm{~s}, 3 \mathrm{H}), 2.97(\mathrm{~s}, 3 \mathrm{H})$, $2.15(\mathrm{dq}, J=6.6,6.1 \mathrm{~Hz}, 2 \mathrm{H}) ;{ }^{13} \mathrm{C}$ NMR $\left(100 \mathrm{MHz}, \mathrm{CD}_{3} \mathrm{OD}\right) \delta$ $176.3,176.1,154.7,136.2,136.1,133.3,133.3,131.9,131.7,128.2$, $128.1,71.2,59.5,59.4,48.8,46.9,34.7,11.2$; HR-MS (ESI+) calcd for $\mathrm{C}_{18} \mathrm{H}_{21} \mathrm{~N}_{2} \mathrm{O}_{4}\left[\mathrm{M}-\mathrm{Br}^{-}\right]^{+}$329.1496, found 329.1493 .

3-(4-Hydroxybutyl)-1-(2-methoxyethyl)-2-methyl-4,9-dioxo4,9-dihydro-1H-naphtho[2,3-d]imidazol-3-ium

bromide (5t)

${ }^{1} \mathrm{H}$ NMR (300 MHz, CD 3 OD) $\delta 8.30-8.23(\mathrm{~m}, 2 \mathrm{H}), 7.97-7.93(\mathrm{~m}$, $2 \mathrm{H}), 4.91(\mathrm{t}, J=4.8 \mathrm{~Hz}, 2 \mathrm{H}), 4.75(\mathrm{t}, J=7.8 \mathrm{~Hz}, 2 \mathrm{H}), 3.83(\mathrm{t}$, $J=4.9 \mathrm{~Hz}, 2 \mathrm{H}), 3.65(\mathrm{t}, J=6.2 \mathrm{~Hz}, 2 \mathrm{H}), 3.33(\mathrm{~s}, 3 \mathrm{H}), 2.95(\mathrm{~s}$, $3 \mathrm{H}), 2.04-1.94(\mathrm{~m}, 2 \mathrm{H}), 1.74-1.65(\mathrm{~m}, 2 \mathrm{H}) ;{ }^{13} \mathrm{C} \mathrm{NMR}(75 \mathrm{MHz}$, $\left.\mathrm{CD}_{3} \mathrm{OD}\right) \delta 176.3,176.1,154.3,136.2,136.2,133.3,131.8,128.2$, $128.2,71.2,62.1,59.4,49.2,30.1,27.3,11.2$; HR-MS (ESI+) calcd for $\mathrm{C}_{19} \mathrm{H}_{23} \mathrm{~N}_{2} \mathrm{O}_{4}\left[\mathrm{M}-\mathrm{Br}^{-}\right]^{+}$343.1652, found 343.1658.

\section{3-(5-Hydroxypentyl)-1-(2-methoxyethyl)-2-methyl-4,9-} dioxo-4,9-dihydro-1H-naphtho[2,3-d]imidazol-3-ium bromide (5u)

${ }^{1} \mathrm{H}$ NMR (800 MHz, DMSO- $\left.\mathrm{d}_{6}\right) \delta 8.20-8.18(\mathrm{~m}, 2 \mathrm{H}), 8.01-8.00$ $(\mathrm{m}, 2 \mathrm{H}), 4.84(\mathrm{t}, J=4.9 \mathrm{~Hz}, 2 \mathrm{H}), 4.65(\mathrm{t}, J=7.6 \mathrm{~Hz}, 2 \mathrm{H}), 4.44$ $(\mathrm{t}, J=5.2 \mathrm{~Hz}, 1 \mathrm{H}), 3.78(\mathrm{t}, J=5.0 \mathrm{~Hz}, 2 \mathrm{H}), 3.40(\mathrm{td}, J=6.2$, $5.4 \mathrm{~Hz}, 2 \mathrm{H}), 3.25(\mathrm{~s}, 3 \mathrm{H}), 2.93(\mathrm{~s}, 3 \mathrm{H}), 1.81$ (quint, $J=7.7 \mathrm{~Hz}$, $2 \mathrm{H}), 1.50-1.46(\mathrm{~m}, 2 \mathrm{H}), 1.42-1.39(\mathrm{~m}, 2 \mathrm{H}) ;{ }^{13} \mathrm{C} \mathrm{NMR}(200 \mathrm{MHz}$, DMSO- $\left.\mathrm{d}_{6}\right) \delta 174.8,174.6,152.7,135.2,135.2,131.6,131.6,130.2$, 130.0, 126.9, 126.8, 69.6, 60.4, 58.5, 47.3, 31.9, 28.7, 22.3, 10.4; LR-MS (FAB+) $m / z 357\left[\mathrm{M}-\mathrm{Br}^{-}\right]^{+}$; HR-MS (FAB+) calcd for $\mathrm{C}_{20} \mathrm{H}_{25} \mathrm{~N}_{2} \mathrm{O}_{4}\left[\mathrm{M}-\mathrm{Br}^{-}\right]^{+}$357.1814, found 357.1818.

3-(6-hydroxyhexyl)-1-(2-methoxyethyl)-2-methyl-4,9-dioxo4,9-dihydro-1H-naphtho[2,3-d]imidazol-3-ium

bromide (5v)

${ }^{1} \mathrm{H}$ NMR (500 MHz, CD 3 OD) $\delta 8.25-8.22(\mathrm{~m}, 2 \mathrm{H}), 7.93-7.91(\mathrm{~m}$, $2 \mathrm{H}), 4.88(\mathrm{t}, J=4.9 \mathrm{~Hz}, 2 \mathrm{H}), 4.68(\mathrm{t}, J=8.0 \mathrm{~Hz}, 2 \mathrm{H}), 3.84(\mathrm{t}, J=$ $6.6 \mathrm{~Hz}, 2 \mathrm{H}), 3.30(\mathrm{~s}, 3 \mathrm{H}), 2.90(\mathrm{~s}, 3 \mathrm{H}), 1.90$ (quint, $J=7.2 \mathrm{~Hz}, 2 \mathrm{H}$ ), 1.56 (quint, $J=6.9 \mathrm{~Hz}, 2 \mathrm{H}), 1.51-1.45(\mathrm{~m}, 4 \mathrm{H}) ;{ }^{13} \mathrm{C} \mathrm{NMR}(125$ $\left.\mathrm{MHz}, \mathrm{CD}_{3} \mathrm{OD}\right) \delta 174.9,174.7,153.0,134.8,134.8,132.0,132.0$, $130.4,126.8,126.8,69.9,61.3,58.0,32.0,29.1,25.8,25.0,9.6$.
3-(2-Aminoethyl)-1-(2-methoxyethyl)-2-methyl-4,9-dioxo4,9-dihydro-1H-naphtho[2,3-d]imidazol-3-ium bromide (5w)

${ }^{1} \mathrm{H}$ NMR (500 MHz, DMSO-d 6 ) $\delta 8.18-8.16$ (m, 2H), 8.10 (bs, $3 \mathrm{H}), 8.00-7.98(\mathrm{~m}, 2 \mathrm{H}), 4.84(\mathrm{t}, J=4.9 \mathrm{~Hz}, 2 \mathrm{H}), 4.83(\mathrm{t}, J$ $=4.1 \mathrm{~Hz}, 2 \mathrm{H}), 3.74(\mathrm{t}, J=4.7 \mathrm{~Hz}, 2 \mathrm{H}), 3.36(\mathrm{~m}, 2 \mathrm{H}), 3.22$ (s, 3H), $2.91(\mathrm{~s}, 3 \mathrm{H})$.

\section{1-(3-Aminopropyl)-3-(2-methoxyethyl)-4,9-dioxo-4,9-} dihydro-1H-naphtho[2,3-d]imidazol-3-ium bromide $(5 x)$

${ }^{1} \mathrm{H}$ NMR (500 MHz, DMSO-d 6 ) $\delta 8.17-8.13$ (m, 2H), 8.00-7.95 (m, 2H), 7.87 (bs, $2 \mathrm{H}), 4.81-78(\mathrm{~m}, 2 \mathrm{H}), 4.68(\mathrm{t}, J=7.7 \mathrm{~Hz}, 1.4 \mathrm{H})$, $4.62(\mathrm{t}, J=7.5 \mathrm{~Hz}, 1.4 \mathrm{H}), 3.75-3.71(\mathrm{~m}, 2 \mathrm{H}), 3.22(\mathrm{~s}, 3 \mathrm{H}), 3.12(\mathrm{q}$, $J=6.1 \mathrm{~Hz}, 0.6 \mathrm{H}), 2.97-2.93(\mathrm{~m}, 1.4 \mathrm{H}), 2.88(\mathrm{~s}, 3 \mathrm{H}), 2.08$ (quint, $J=7.6 \mathrm{~Hz}, 1.4 \mathrm{H}), 1.91(\mathrm{t}, J=7.4 \mathrm{~Hz}, 0.6 \mathrm{H})$.

\section{1,3-Bis(2-methoxyethyl)-4,9-dioxo-4,9-dihydro-1H-}

naphtho[2,3-d]imidazol-3-ium bromide (5y)

${ }^{1} \mathrm{H}$ NMR (300 MHz, CD $\left.3 \mathrm{OD}\right) \delta 8.27(\mathrm{dd}, J=5.8,3.4 \mathrm{~Hz}, 2 \mathrm{H})$, $7.94(\mathrm{dd}, J=5.9,3.3 \mathrm{~Hz}, 2 \mathrm{H}), 4.92(\mathrm{t}, J=4.8 \mathrm{~Hz}, 4 \mathrm{H}), 3.85(\mathrm{t}$, $J=4.8 \mathrm{~Hz}, 4 \mathrm{H}), 3.31(\mathrm{~s}, 6 \mathrm{H}), 2.91(\mathrm{~s}, 3 \mathrm{H}) ;{ }^{13} \mathrm{C} \mathrm{NMR}(75 \mathrm{MHz}$, $\left.\mathrm{CD}_{3} \mathrm{OD}\right) \delta 176.3,155.7,136.2,133.3,131.7,128.2,71.3,59.4$, 49.2, 11.5; HR-MS (ESI+) calcd for $\mathrm{C}_{19} \mathrm{H}_{23} \mathrm{~N}_{2} \mathrm{O}_{4}\left[\mathrm{M}-\mathrm{Br}^{-}\right]^{+}$ 392.1496, found 392.1504.

\section{1-Butyl-3-(2-methoxyethyl)-4,9-dioxo-4,9-dihydro-1H-}

naphtho[2,3-d]imidazol-3-ium bromide (5z)

${ }^{1} \mathrm{H}$ NMR (800 MHz, CD 3 OD) $\delta 8.27-8.25(\mathrm{~m}, 2 \mathrm{H}), 7.95-7.94(\mathrm{~m}$, $2 \mathrm{H}), 4.90(\mathrm{t}, J=4.9 \mathrm{~Hz}, 2 \mathrm{H}), 4.71(\mathrm{t}, J=7.9 \mathrm{~Hz}, 2 \mathrm{H}), 3.86(\mathrm{t}$, $J=4.9 \mathrm{~Hz}, 2 \mathrm{H}), 3.33(\mathrm{~s}, 3 \mathrm{H}), 2.92(\mathrm{~s}, 3 \mathrm{H}), 1.92-1.86(\mathrm{~m}, 2 \mathrm{H})$, 1.53 (sext, $J=7.5 \mathrm{~Hz}, 2 \mathrm{H}), 1.04(\mathrm{t}, J=7.4 \mathrm{~Hz}, 3 \mathrm{H}) ;{ }^{13} \mathrm{C} \mathrm{NMR}$ $\left(200 \mathrm{MHz}, \mathrm{CD}_{3} \mathrm{OD}\right) \delta 176.3,176.1,154.3,136.2,136.2,133.4$, 133.3, 131.7, 131.7, 128.2, 128.2, 71.2, 59.4, 49.2, 48.9, 32.4, 20.7, 13.9, 11.0; HR-MS (ESI+) calcd for $\mathrm{C}_{19} \mathrm{H}_{23} \mathrm{~N}_{2} \mathrm{O}_{3}\left[\mathrm{M}-\mathrm{Br}^{-}\right]^{+}$ 327.1706, found 327.1703.

\section{hPSCs and Differentiated Cell Culture}

Human ESCs (H9, Wicell Research Institute) and hiPSCs (SES8) (Lee et al., 2010) were maintained in ESC medium (Stem Cell Technology, \# 29106). For treatment of YM155 and YM155 analogs, hESCs or hiPSCs were cultured in mTeSR 1 medium (with $50 \times$ supplement, $0.1 \%$ gentamycin) on Matrigel (Corning, \# 354277)-coated $60 \mathrm{~mm}$ tissue culture dishes (Corning, \# 430166) or tissue culture 6, 12, 24-well plate (Falcon, \# 353046, \# 353043, \# 353047), as described previously (Cho et al., 2015, 2016, 2018; Jeong et al., 2017b; Kim S. Y. et al., 2017). Smooth muscle cells (hASMC and SMC3) (Lee et al., 2010) were cultured in SMCM medium (ScienCell Research Laboratories, \# 1101).

\section{Cell Death Assay}

For Annexin V staining, cells with YM155 or YM155 analogs treated for $24 \mathrm{~h}$, were washed twice with PBS and were stained with FITC-Annexin V (BD Bioscience, \# 556419) and 7-AAD (BD Bioscience, \# 559925) or PE-Annexin V (BD Bioscience, \# 556421) and 7-AAD for $30 \mathrm{~min}$ at $\mathrm{RT}$ in 
the dark. Cell death was analyzed with FACS as described previously (BD Bioscience) (Kwon et al., 2017). Cells stained with Annexin / 7-AAD were analyzed by FACS Calibur I (BD Bioscience). For all of the cell images captured, Light channel of optical microscope (Olympus, CKX-41) or JULI-stage (NanoEntek) were used according to the manufacture's protocol.

\section{Immunoblotting Assay}

Immunoblotting assay were performed as described previously (Kwon et al., 2017). Antibodies used in this study, PARP-1 (\# sc7150 ) and $\alpha$-tubulin (\# sc-8035), were purchased from Santa Cruz Biotechnology. Antibodies for cleaved caspase-3 (\#9664S) were purchased from Cell Signaling Biotechnology.

\section{Structure Optimization and Electrostatic Surface Potential Analysis}

MarvinSketch was used for drawing, displaying and characterizing chemical structures, substructures, and reactions, MarvinSketch 17.28, 2018, ChemAxon (http://www.chemaxon. com). The YM155 and its analogs were prepared in the form of 3D structures with Chem3D software (http://www.perkinelmer. com) that immediately converted the $2 \mathrm{D}$ chemical structures into $3 \mathrm{D}$ structures. Initially, energy minimization of $3 \mathrm{D}$ structures was done with MM2 force field in Chem3D. For further refinement, minimization was performed with UFF/GAFF force field in Avogadro (Hanwell et al., 2012). The Coulombic surface coloring method with UCSF Chimera was used to calculate the electrostatic surface potentials of the YM155 analogs. All structures were represented with UCSF Chimera (Pettersen et al., 2004; Goddard et al., 2005).

\section{Statistical Analysis}

The graphical data were shown as mean \pm SEM. Statistical significance between two or three groups were determined by using ordinary one-way or two-way ANOVA after Bonferroni posttest, respectively. Significance was assumed for $P<0.05\left(^{*}\right), 0.01\left(^{* *}\right), 0.001\left({ }^{* *}\right)$ and $0.0001(* * *)$.

\section{RESULTS AND DISCUSSIONS}

\section{Synthesis of YM Analogs and Requirement of the Imidazolium Ring Structure for Stemotoxic Activity}

Naphthoquinone imidazolium YM155 analogs were synthesized based on the procedure shown in Scheme 1. Dichloronaphthoquinone (1) was reacted with 2methoxyethylamine in the presence of triethylamine to afford 2 , and subsequently acetylated to afford $\mathbf{3}$. Reaction of $\mathbf{3}$ with diverse amines afforded $\mathbf{4}$, and compounds were cyclized in the presence of $\mathrm{HBr}$ in refluxing $\mathrm{EtOH}$ to afford naphthoquinone imidazolium salt 5. Compound $\mathbf{6}$ was synthesized according to the procedure reported previously (Kuo et al., 1996). Structures of synthesized compounds are listed in Table 1. Through initial screening of 30 YM155 analogs based on cellular morphology, YM155 analogs with stemotoxic activity were selected (Figure S1). In particular, loss of the pyrazine moiety of YM155 (shown as 6) results in the clear reduction of stemotoxic activity toward hESCs based on cellular morphology (Figure 1A). The significance of pyrazine moiety of YM155 in stemotoxic activity was confirmed by the level of active caspase 3 formation (Figures 1B-D). Among the first set of YM155 analogs, two YM155 analogs (5r and 5s) with hydroxyl groups induced cell death in hESCs, which was most pronounced with 5s (Figure S1; Figure 1E). It is noteworthy that YM155 analogs with uncyclized moieties (4), unsubstituted benzyl groups (5c), and substituted phenyl groups $(\mathbf{5 a}, \mathbf{5 b})$ showed no activity (Figure S1; Figure 1E).

\section{Hydrogen Bond Acceptor Distance Markedly Affects Stemotoxic Activity}

Given that 5s, possessing a 3-hydroxypropyl group, appeared more potent than 5r, possessing a 2-hydroxyethyl group (Figure 1E), we altered the carbon chain harboring the hydroxyl group and examined the stemotoxic activity. Using this approach, we synthesized three more YM155 analogs with carbon chains of differing length (from two to six). As shown in Figure 2B, stemotoxic activity was dramatically attenuated in YM155 analogs with more than three carbons in the chain, suggesting that the presence of the hydroxyl group at a certain distance from the imidazolium ring structure may be important for stemotoxic activity. Interestingly, YM155 analogs, with an amine group (5w and $\mathbf{5 x}$ ) instead of a hydroxyl group at the same distance (Figure S2), displayed only weak or no stemotoxic activity. Given that the nitrogen atom in amine groups is a much weaker hydrogen bond acceptor than the oxygen atom, this result is consistent with our hypothesis. Additionally, YM155 analogs with ammonium salts present as protonated forms at physiological $\mathrm{pH}$ and can in principle serve as hydrogen bond donors rather than hydrogen bond acceptors, owing to the high pKa value $(\sim 10-11)$ of the conjugate acids of aliphatic amine groups when present at the same distance as hydroxyl groups. Accordingly, we hypothesized that the presence of a hydrogen bond acceptor at a specific distance may be crucial for stemotoxic activity. Next, we examined whether $\mathbf{5 s}$ retained stemotoxic activity by screening against isogenic human aortic smooth muscle cells (HASMCs), iPSCs from HASMCs (SES8 cells), and SMCs derived from SES8 cells (SMC3 cells), as described previously (Lee et al., 2013). Similar to YM155, 5s induced selective cell death of human iPSCs (SES8 cells) in a dose-dependent manner, but not differentiated smooth muscle cells (HASMCs or SMC3s; Figure 2D), although stemotoxic activity was weaker than that of YM155.

\section{Nitrogen in the Imidazolium Ring of YM155 Is a Hydrogen Bond Acceptor, and Crucial for Stemotoxic Activity}

Since the hydroxyl group in $\mathbf{5 s}$ is located three carbons away from the imidazolium moiety, and is a putative hydrogen bond acceptor important for stemotoxic activity, we speculated that one of the two nitrogens in the pyrazine ring of YM155 may serve as a hydrogen bond acceptor and may also contribute 


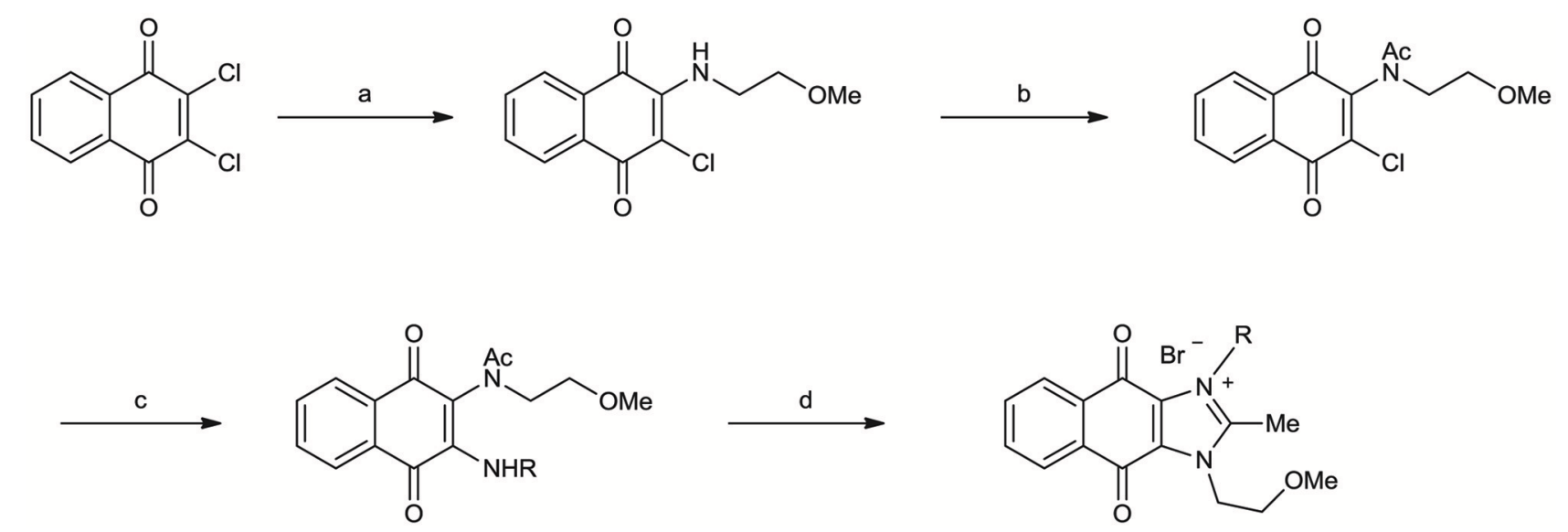

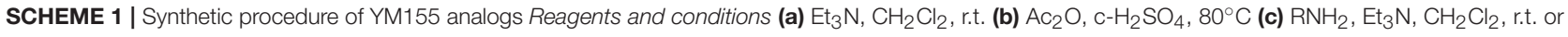
$\mathrm{CHCl}_{3}$, reflux (for $\left.\mathbf{5 a}, \mathbf{5 b}\right)$ (d) $\mathrm{HBr}$, EtOH, reflux.

TABLE 1 | Structures of synthesized imidazolium compounds.<smiles>[R][N+]1(Br)C2=C(C(=O)c3ccccc3C2=O)[N+](CCOC)=C1C</smiles>

\begin{tabular}{|c|c|c|c|c|c|c|c|c|}
\hline $5 a$ & & - & $5 j$ & & + & $5 s$ & & +++ \\
\hline $5 b$ & & - & $5 k$ & & + & $5 t$ & & + \\
\hline $5 d$ & & + & $5 m$ & & +++ & $5 v$ & & + \\
\hline $5 \mathrm{~g}$ & & - & $5 p$ & & + & $5 y$ & & + \\
\hline $5 \mathrm{~h}$ & & - & $5 q$ & & - & $5 z$ & & - \\
\hline $5 i$ & & - & $5 r$ & & ++ & & & \\
\hline
\end{tabular}


A
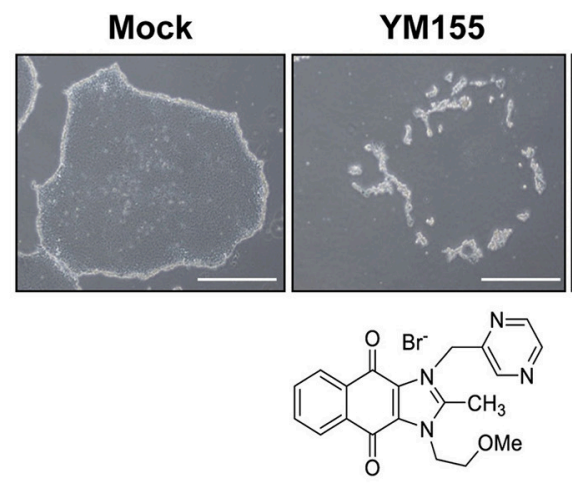

B
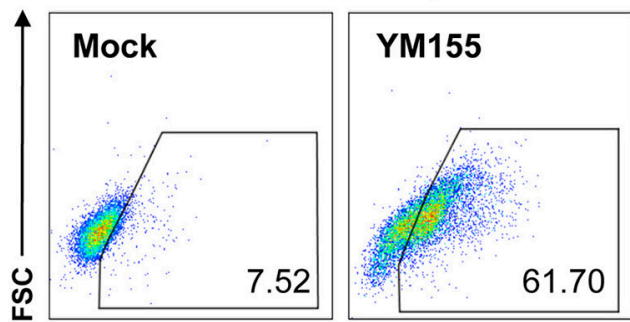

C.Casp3 (FL-1)
C

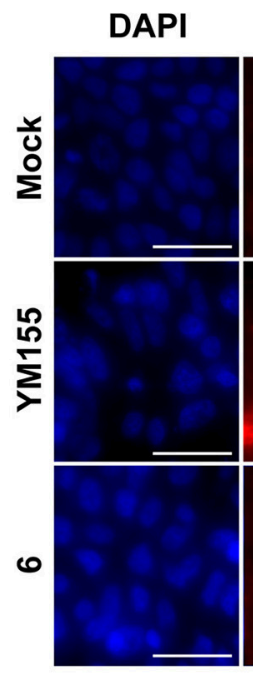

\section{C.Casp3}

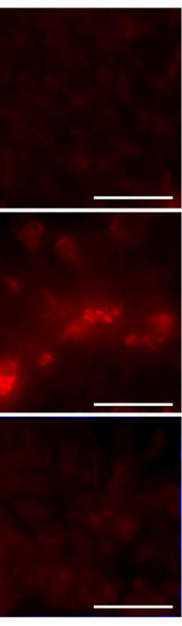

MERGED

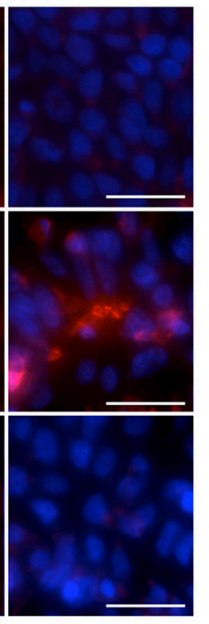

6
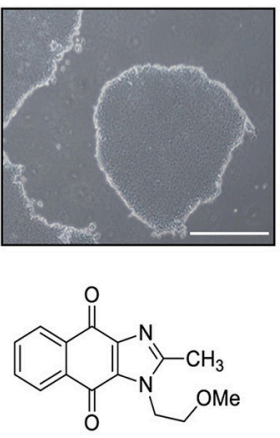

6

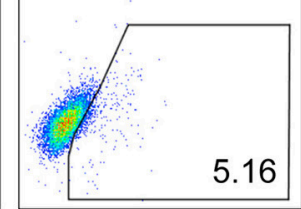

5.16

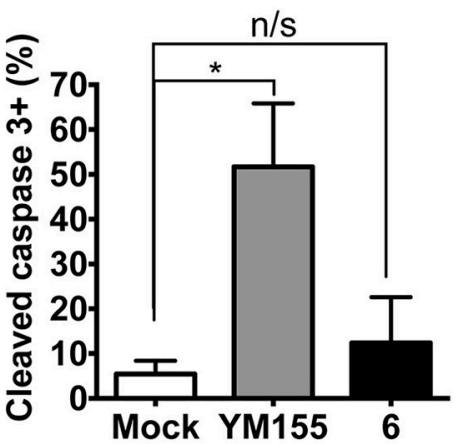

D

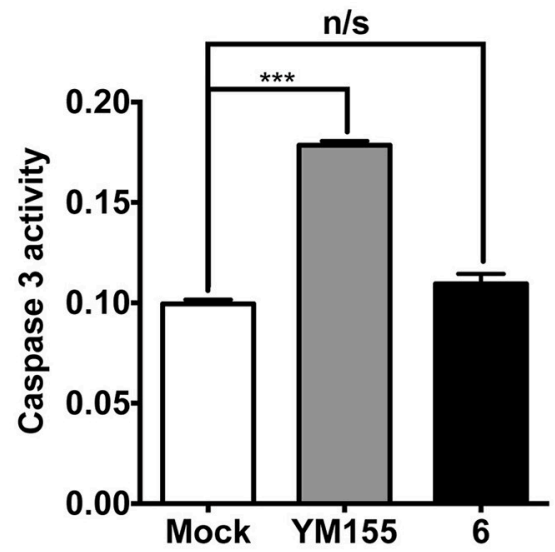

E

\begin{tabular}{llllll} 
Code & $5 \mathrm{r}$ & $5 \mathrm{~s}$ & $4 \mathrm{r}$ & $5 \mathrm{c}$ & $5 \mathrm{a}$ \\
\hline
\end{tabular}
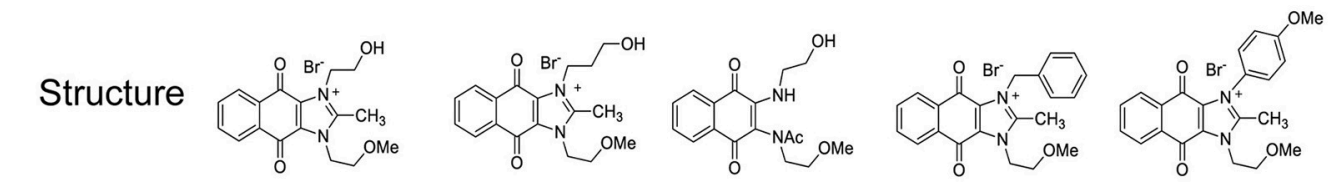

Activity

$+$

$++$

FIGURE 1 | Requirement of the imidazolium ring structure for stemotoxic activity (A) Light microscopic images of hiPSCs after treatment of YM155 (50 nM) and $\mathbf{6}$ (50 nM) for $24 \mathrm{~h}$ (top), Structure of YM155 and 6 (bottom) (Scale bar = $300 \mu \mathrm{m})$. (B) FACS analysis for the cleaved caspase3 (C.Casp3) staining (left), Percentage of cleaved caspase 3 positive cells were presented as bar graph (right) $(n=3)\left(P<0.05\left(^{*}\right)\right)$. (C) Fluorescence microscopic images of hiPSCs $24 \mathrm{~h}$ after $50 \mathrm{nM}$ of YM155 or 6, stained for cleaved caspase3 (C.Casp3) with DAPI nuclear counterstaining (Scale bar $=50 \mu \mathrm{m}$ ). (D) Relative enzyme activity of caspase3 after treatment of indicated chemicals $(n=3)\left(P<0.05\left(^{(* \star}\right)\right)(\mathbf{E})$ Summary table of three YM155 analogs with chemical structures. 


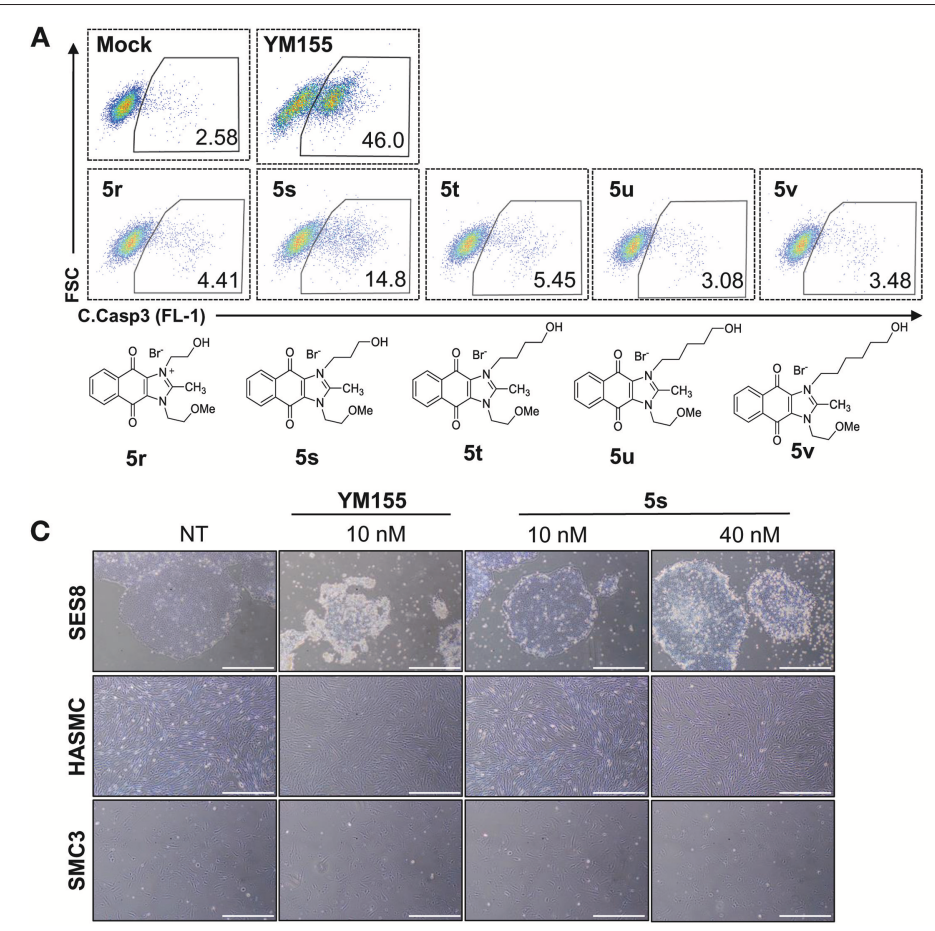

B
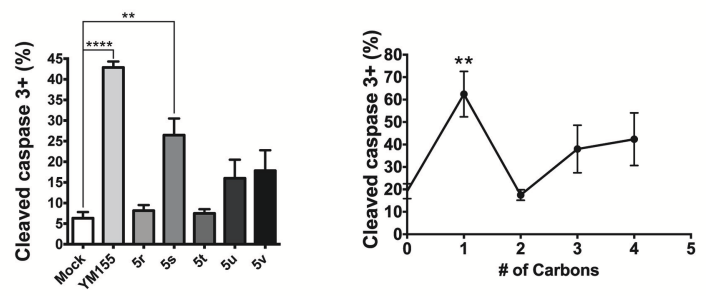

D

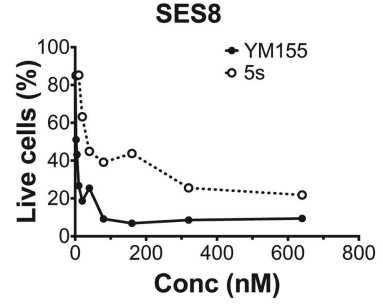

SMC3

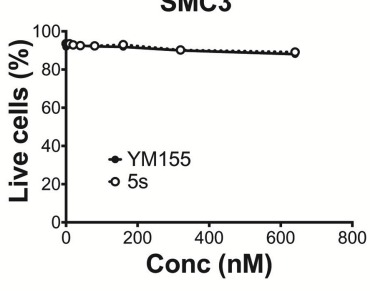

FIGURE 2 | Hydrogen bond acceptor distance markedly affects stemotoxic activity (A) FACS analysis for cleaved caspase3 after treatment of YM155 and YM155 analogs (50 nM), Chemical structures of YM155 analogs were presented below. (B) Percentage of cleaved caspase3-positive cells at indicative YM155 analogs (left) and number of carbon chain of alcohol group of each compound (right) $(n=4)$ was presented as bar graph $\left(P<0.01\left({ }^{* *}\right), 0.0001\right.$ (***)). (C) Light microscopic images of hiPSC and differentiated cells (HASMC and SMC3) $24 \mathrm{~h}$ after treatment of indicated compounds (Scale bar $=300 \mu \mathrm{m}$ ). (D) Percentages of live cells (Annexin-V negative and 7-AAD negative population) after treatment of YM155 and $\mathbf{5 s}$ in hiPSCs (SES8: left) and SMC3 (right) were presented.

to stemotoxic activity. To test this, 5c lacking the two nitrogens in the aromatic ring was prepared, and as expected, stemotoxic activity was completely lost (Figure 1E). Thus, we next synthesized three more YM155 analogs with nitrogen located at different positions (ortho, meta, and para) of the aromatic ring, and tested the effect on hESCs. Strikingly, $5 \mathbf{l}$ with one nitrogen atom in the meta position exhibited greater stemotoxic activity than 5s, with a 3-hydroxylpropyl group serving as a hydrogen bond acceptor (Figure S3A). As shown in Figure $\mathbf{3 A}, 5 \mathrm{~m}$ with a nitrogen in the para position of the aromatic ring was more effective toward hESCs (Figure 3A) than a nitrogen in the ortho (5k) or meta (5l) positions (Figure S3B). We noticed that both $5 \mathbf{l}$ (meta) and $5 \mathrm{~m}$ (para) induced cell death of hESCs comparably to YM155 when the dose was $>10 \mathrm{nM}$ (Figure 3B). However, $5 \mathrm{~m}$ appeared to be more potent than 51 below the $5 \mathrm{nM}$ range, and equivalent to YM155 (Figure 3C).

\section{Stemotoxic Activity of $\mathbf{5 m}$}

To safely apply stemotoxic agents that ablate undifferentiated hPSCs, differentiated cells derived from hPSCs should remain unaffected after treatment, while undifferentiated hPSCs should undergo selective cell death (Jeong et al., 2017a). To test this, we examined the reactivity of YM155 analogs showing moderate to high stemotoxic activity (e.g., $5 \mathbf{l}$ and $5 \mathrm{~m}$ ) toward differentiated cells derived from hPSCs. As shown in Figures 4A,B, both $5 \mathrm{l}$ (Figure 4A) and 5m (Figure 4B) induced cell death only in SSEA3-positive populations (i.e., undifferentiated hPSCs) but not SSEA3-negative populations (differentiated cells derived from hPSCs) when used to treat partially differentiated hPSCs (a mixture of differentiated and undifferentiated cells). Furthermore, the functionality of SMCs derived from iPSCs (Lee et al., 2010, 2013), determined by $\mathrm{Ca}^{2+}$ influx (Lee et al., 2010), remained unaltered after treatment with $\mathbf{5 s}$ or $\mathbf{5 m}$, similar to YM155 (Figure 4C). These results clearly suggest that $\mathbf{5 m}$ may be applicable as a stemotoxic compound.

\section{Hydrogen Bond Acceptors Strongly Influence the Stemotoxic Activity of YM155 Analogs}

Given that $\mathbf{5 m}$ with a nitrogen at the para position showed stemotoxic activity equivalent to YM155, we speculated that a hydrogen bond acceptor at that position may be important for interaction with the target protein, and hence may be critical for stemotoxic activity. To test this, we synthesized six YM155 analogs with hydrogen bond acceptors at different positions on the benzene ring (Figure 5A), and screened them against hPSCs. As shown in Figures 5B,C, 5f, which mimics $5 \mathrm{~m}$ and has a hydrogen bond acceptor at the para position, displayed clear stemotoxicity, while 5d (mimicking 


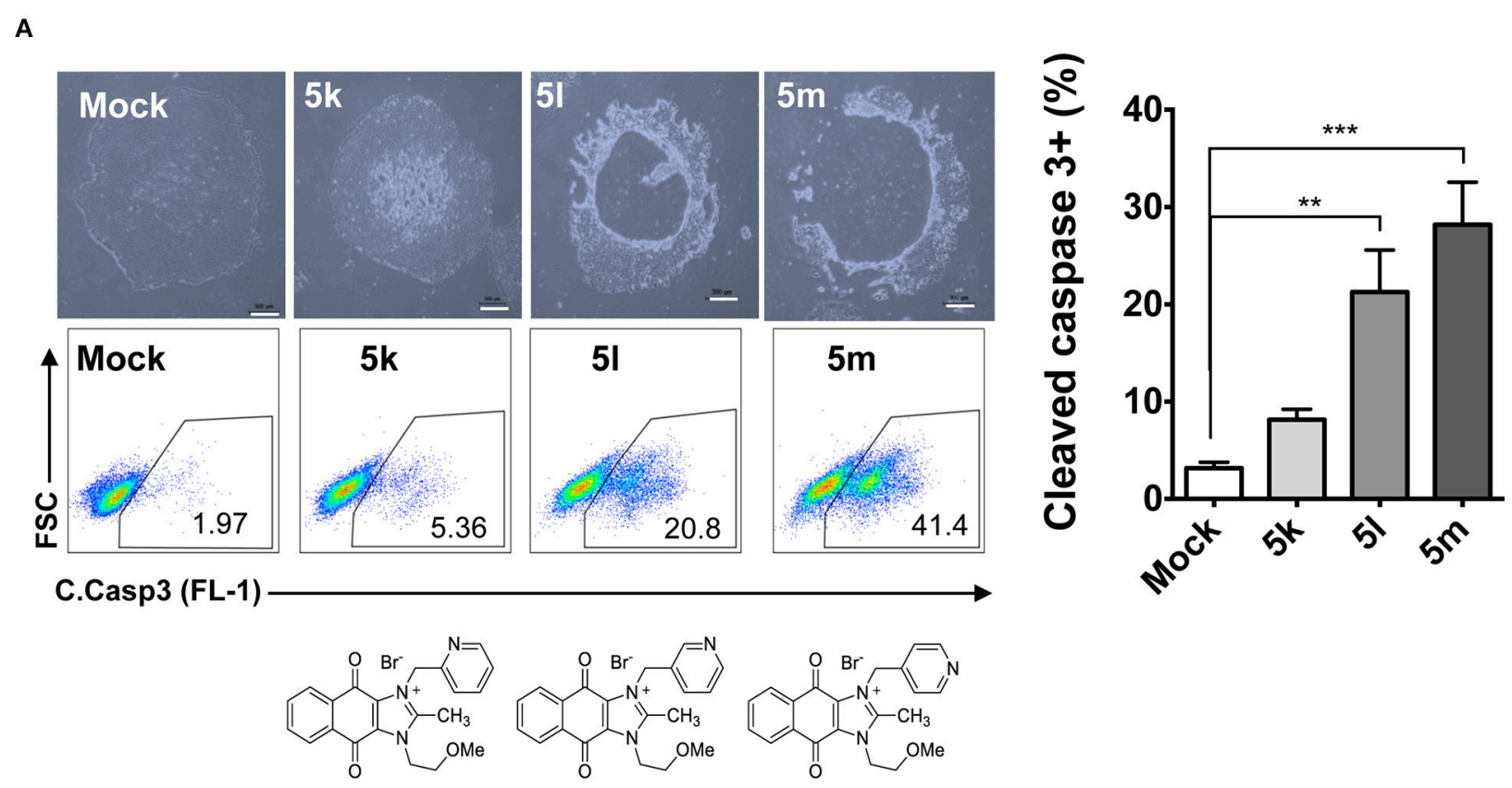

B

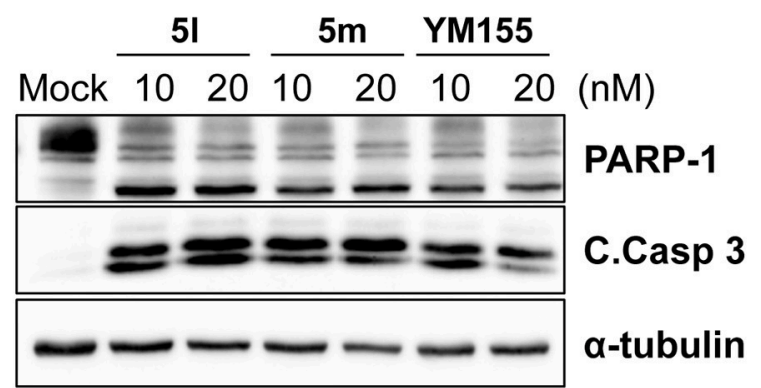

C

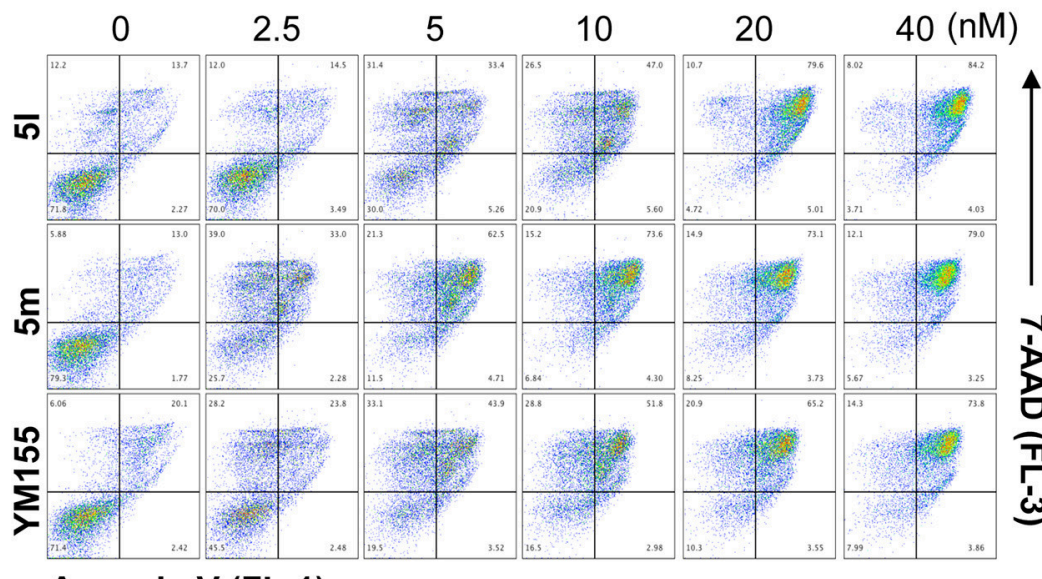

Annexin V (FL-1)

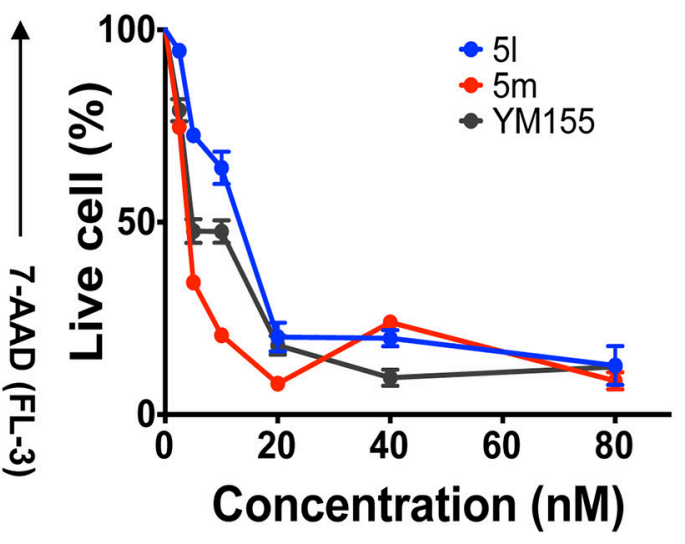

FIGURE 3 | Nitrogen in the pyrazine ring of YM155 is a hydrogen bond acceptor, and crucial for stemotoxic activity (A) Light microscopic images (top) and FACS analysis for cleaved caspase3 staining after treatment of indicated compounds $(\mathbf{5 k}, \mathbf{5 1}$, and $\mathbf{5 m}, 50 \mathrm{nM})(\mathrm{bottom})$ with chemical structure, Percentages of cleaved caspase3 positive cells were presented as bar graph (right) $(n=4)$ (Scale bar $\left.=300 \mu \mathrm{m})\left(P<0.01\left(^{* \star}\right), 0.001{ }^{\star \star \star}\right)\right)$. (B) Immunoblotting analysis for PARP-1, cleaved caspase 3 (C.Casp 3) after treatment of indicative dose of compounds, $\alpha$-tubulin for equal protein loading control. (C) FACS analysis for Annexin-V/7-AAD after indicative dose of YM155 and YM155 analogs in hESCs (left), Percentages of live cells were graphically presented (right). 

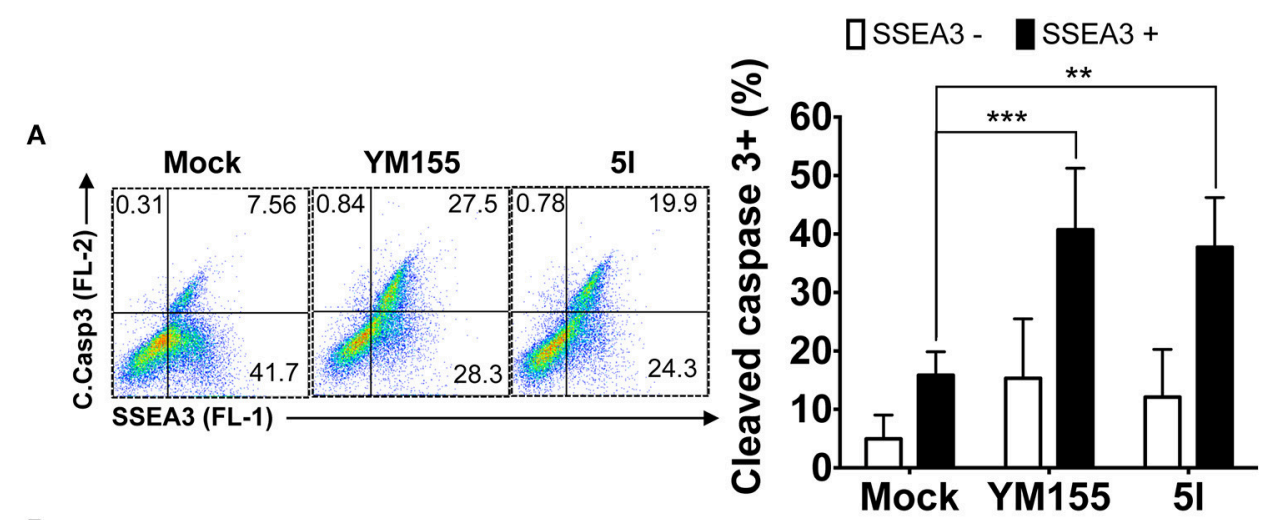

B

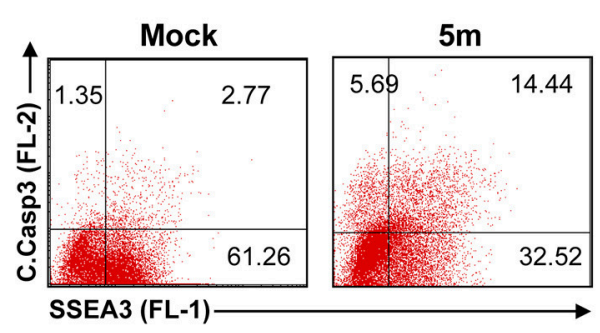

C
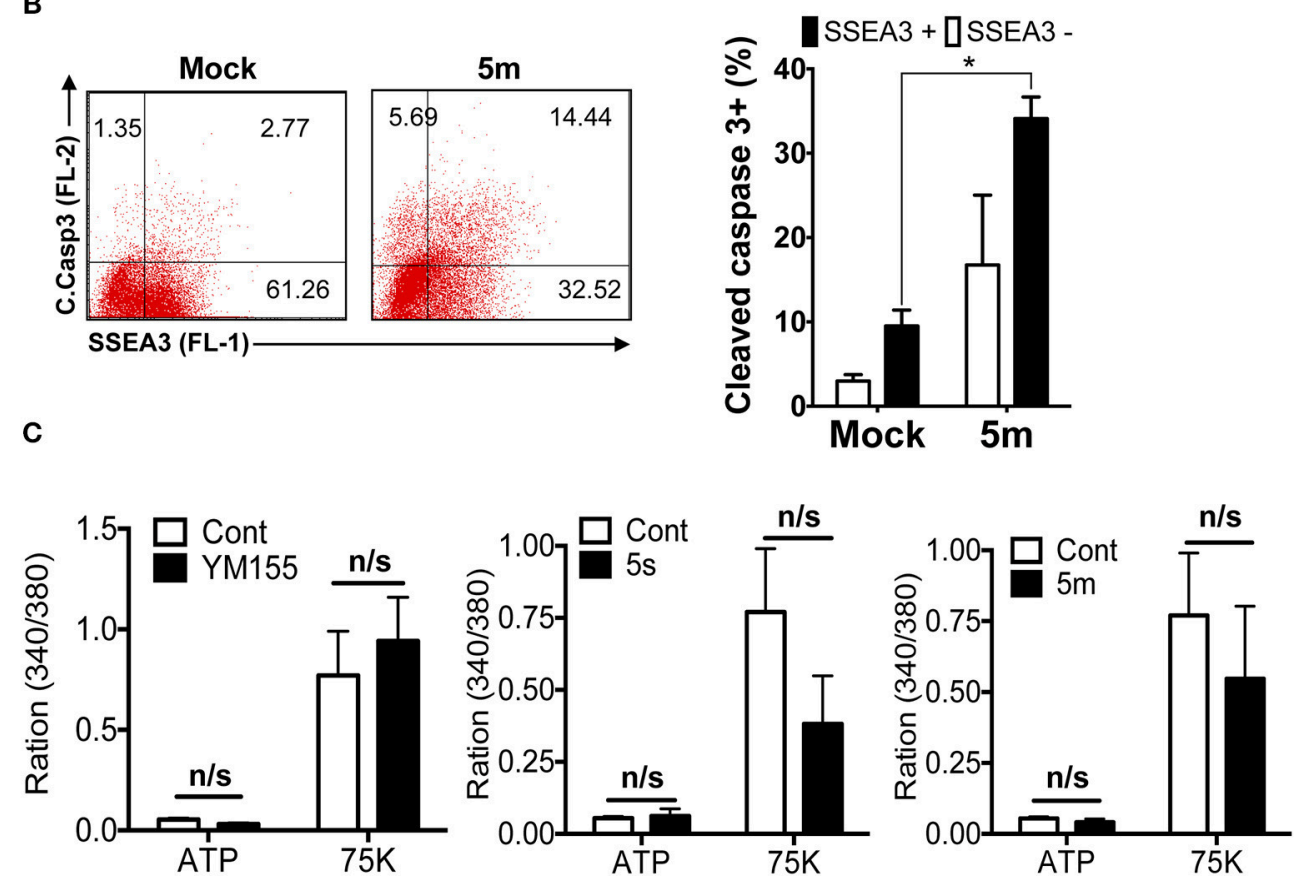

FIGURE 4 | Stemotoxic activity of 5m (A) FACS analysis for cleaved caspase3 and SSEA3 staining after treatment of YM155 or $\mathbf{5 I}$ (left), Percentages of cleaved caspase ${ }^{+}$cells were presented by bar graph (right) $(n=4)\left(P<0.01\left(^{\star \star}\right), 0.001\left(^{\star \star \star *}\right)\right)$. (B) FACS analysis for cleaved caspase3 and SSEA3 staining after treatment of $\mathbf{5 m}$, Percentages of cleaved caspase $3^{+}$cells were presented by bar graph (right) $(n=4)\left(P<0.05\left(^{*}\right)\right)$. (C) Net changes in intracellular calcium levels in response to a pharmacological agonist, ATP $(20 \mu \mathrm{M})$ or membrane depolarization $(75 \mathrm{mM} \mathrm{K+)}$ after treatment of indicated chemicals in the SMC3 cells.

5k) or 5e (mimicking 5l) exhibited only moderate stemotoxic activity (Figures 5B,C). It is noteworthy that each analog with a methoxy group (5h, i, and $\mathbf{j}$ ) lost all activity (Figure 5B). The unexpectedly low stemotoxic activity of $\mathbf{5 h}-\mathbf{j}$, even when a hydrogen bond acceptor (methoxy substituent) was present, is likely due to steric hindrance of the adjacent methyl group, which might interfere with interactions between small molecules and target proteins. Additionally, the results of fluoride substitution, which can also function as a hydrogen bond acceptor rather than a hydrogen bond donor, support this proposal. Compound $\mathbf{5 f}$ showed higher stemotoxic activity than $\mathbf{5 e}$, as determined by cellular morphology (Figure 5C) and immunoblotting of apoptotic markers (PARP1 cleavage and formation of active caspase 3; Figure 5E), suggesting that the location of the hydrogen bond acceptor is critical for stemotoxic activity via recognition by the target protein.

\section{SLC35F2 Is Required for Cell Entry of YM155 Analogs and Hence Stemotoxic Activity}

Based on the role of the solute carrier protein SLC35F2 in the import of YM155 (Winter et al., 2014), and differences in the cytotoxicity of YM155 toward different cancer cell types (Winter et al., 2014), we hypothesized that the stemotoxic activity of YM155 and its analogs may be mediated by SLC35F2. To explore this, cell death of hESCs after treatment with various stemotoxic YM155 analogs was compared with control hESCs 
A<smiles>[R][n+]1c2c(n(CCOC)c1C)C(=O)c1ccccc1C2=O</smiles>

B

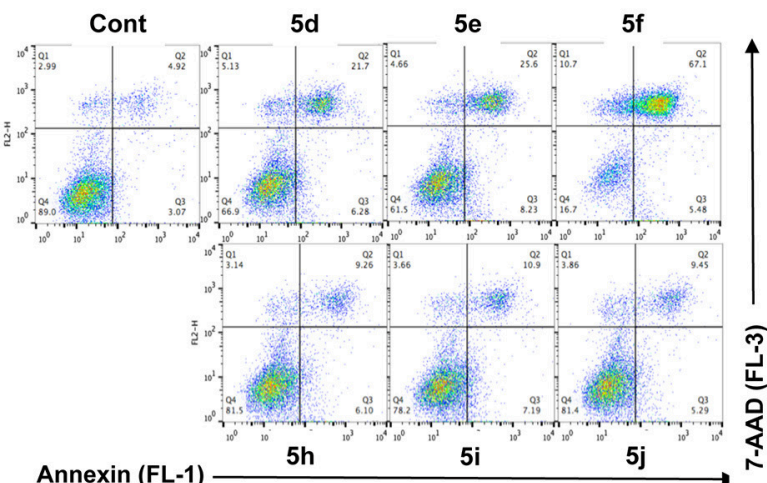

5i 5 j

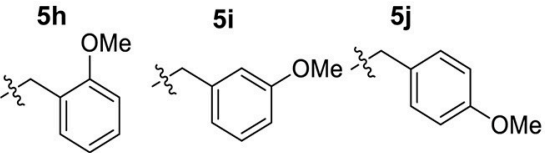<smiles>Fc1cccc(Cc2cc(F)ccc2Cc2cccc(Br)c2)c1</smiles>

ortho meta para

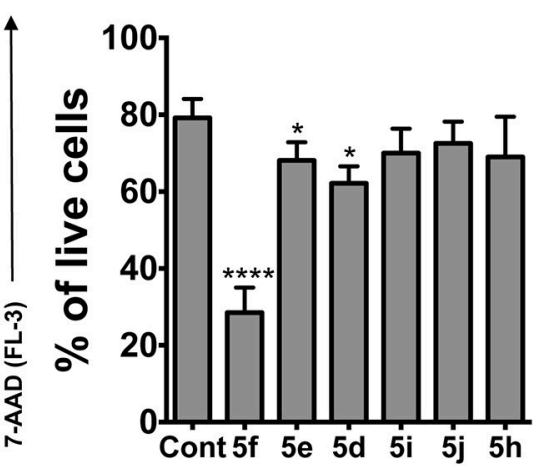

C
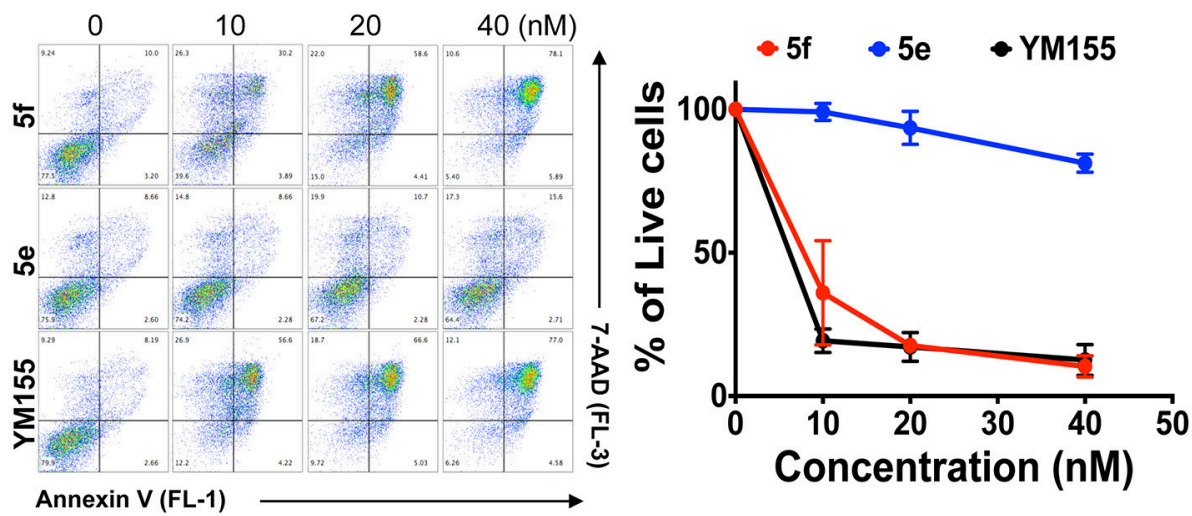

D

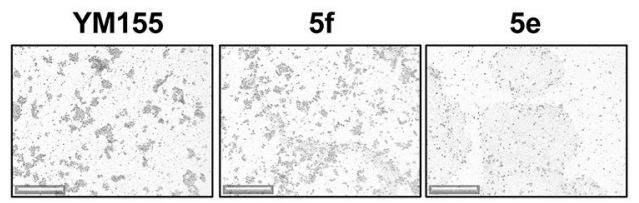

E $\quad 0 \quad 2550 \quad 2550 \quad 2550(n M)$

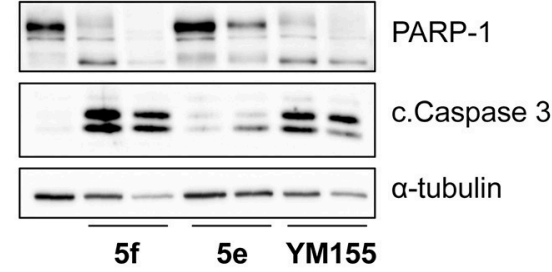

FIGURE 5 | Hydrogen bond acceptors strongly influence the stemotoxic activity of YM155 analogs (A) Chemical backbone structure of YM155 analogs (left) and six YM155 analogs (right). (B) FACS analysis for Annexin-V/PI staining after treatment of indicated compounds, Percentages of live cells (Annexin-V negative and PI negative Cells) were presented as bar graph (right) $(n=6)\left(P<0.05\left(^{\star}\right), 0.0001\left(^{(* \star *)}\right)\right.$. (C) FACS analysis for Annexin-V/7-AAD staining after indicative concentration of YM155 and YM155 analogs (5e or 5f) (left). Percentages of live cells (Annexin-V negative and 7-AAD negative Cells) were presented as bar graph (right). (D) Light microscopic images after treatment of $20 \mathrm{nM}$ of YM155 and YM155 analogs (Scale bar $=500 \mu \mathrm{m}$ ). (E) Immunoblotting analysis for PARP-1 and cleaved caspase 3 (C.Casp3) after indicative concentration of YM155 analogs, $\alpha$-tubulin for equal protein loading control.

(wild type: WT) and SLC35F2 knockout (KO) hESCs generated using the CRISPR/CAS9 system (Figures 6A,B and Figure S4; Kim et al., 2019). As predicted, the stemotoxic activity of
YM155 and its analogs was completely lost in KO hESCs, while cell death was evident in WT hESCs (Figures 6C,D). Considering the pivotal role of SLC35F2 for import of YM155 
A

\section{SLC35F2}

E1 E2 E3 E4 E5 E6 E7 E8

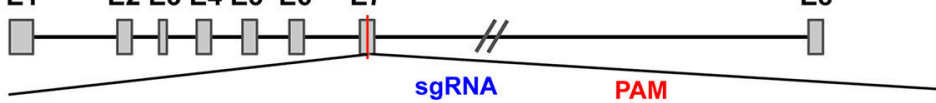

5' - TGGTGATTAAAGTCACTAGTGCCACTTCCGTCAACCTGGGCATCCTGACAG - 3'

3’ - ACCACTAATTTCAGTGATCACGGTGAAGGCAGTTGGACCCTAGGACTGTC - 5'

B

Indel Sequence

WT AAGTCACTAGTGCCACTTCCGTCAA|CCTGGGCATCCTGACAGCGGACCTCTACAGCCTTTTTGTTGGACTCTTTC

-25 AAGTCACTAG--..-CTGACAGCGGACCTCTACAGCCTTTTTGTTGGACTCTTTC

+1 AAGTCACTAGTGCCACTTCCGTCAA |ACCTGGGCATCCTGACAGCGGACCTCTACAGCCTTTTTGTTGGACTCTTT

-3 AAGTCACTAGTGCCACTTCCGT-- - | CCTGGGCATCCTGACAGCGGACCTCTACAGCCTTTTTGTTGGACTCTTTC

C

\begin{tabular}{l|lllll} 
DMSO & YM155 & $5 \mathrm{I}$ & $5 \mathrm{~m}$ & $5 \mathrm{f}$ & $5 \mathrm{e}$
\end{tabular}
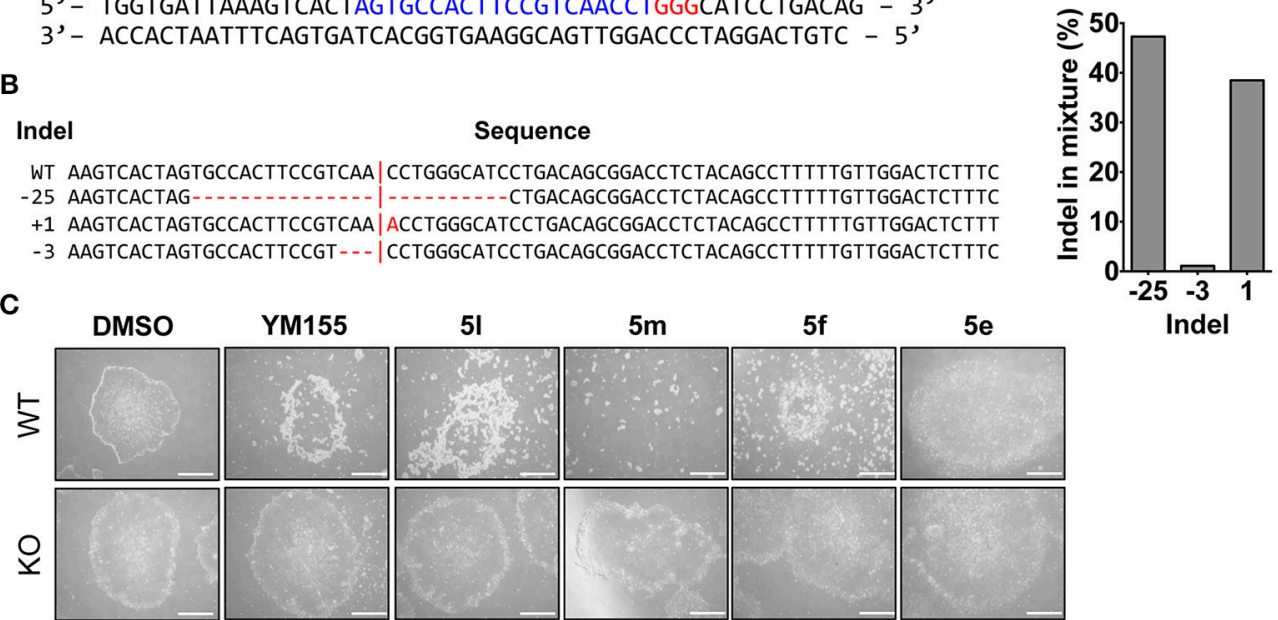

D

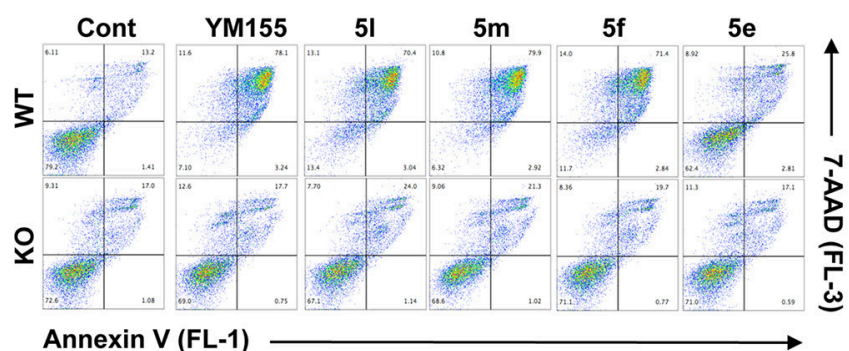

Annexin V (FL-1)

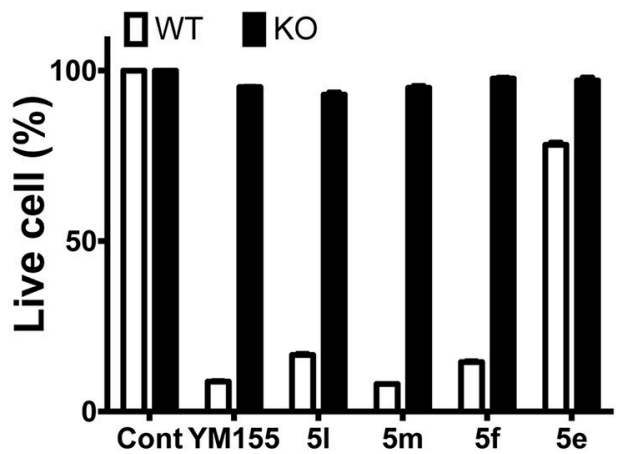

FIGURE 6 | SLC35F2 is required for cell entry of YM155 analogs and stemotoxic activity (A) Scheme for knock out (KO) of SLC35F2 targeting Exon 7. (B) Percentage of mutations quantified from Sanger sequencing and relative contribution of each sequence of wild type (WT) and SLC35F2 KO hESCs was graphically presented.

(C) Light microscope images of hESCs (WT) and SCL35F2 knock out hESCs (KO) after treatment of $20 \mathrm{nM}$ of YM155 and YM155 analogs (Scale bar = $300 \mu \mathrm{m}$ ).

(D) FACS analysis for Annexin-V/7-AAD staining after indicative concentration of YM155 in WT or KO (left), Percentages of live cells (Annexin-V negative and 7-AAD

negative Cells) were presented as a bar graph (right) $(n=3)$.

into cells (Winter et al., 2014), the chemical structure of YM155 analogs (e.g., the position of the hydrogen bond acceptor in the aromatic ring) may be important for interaction with SLC35F2 in hESCs.

\section{Structure-Activity Relationships of YM155 Analogs}

Using Tanimoto (or Jaccard) analysis of molecular similarity (Maggiora et al., 2014), the structural similarity between YM155 
analogs displaying stemotoxic activity and YM155 was compared. Compound $\mathbf{5 f}$ has a similar structure to that of $\mathbf{5 m}$ (Figure $7 \mathbf{A}$ ). Given that the stemotoxic activity of $\mathbf{5 m}$ and $\mathbf{5 f}$ was equivalent to that of YM155 (Figures 3, 5), whereas the stemotoxic activity of $\mathbf{5 l}$ (or $5 \mathbf{k}$ ) and $\mathbf{5 e}$ (or $\mathbf{5 d}$ ) was not, the presence of a hydrogen acceptor (e.g., nitrogen or fluoride) at the para position, and not the ortho or meta position, may be favorable for the formation of hydrogen bonds with the SLC35F2 target protein (Figure 7B). The structure of YM155 can be divided into three parts in 3D space; a dioxonaphtho-imidazolium part occupying the largest space, a pyrazine aromatic ring connected to the imidazolium ion, and a linear methoxyethyl chain also connected to imidazolium ion. A surface model shows that the pyrazine ring and methoxyethyl chains are attached to opposite sides of the flat dioxonaphtho-imidazolium backbone in YM155
(Figure 7B). Comparison of the surface models and activities of YM155 analogs showed that compounds with a shape that differed from that of YM155 were less active than YM155. For instance, the activity of analogs, in which the pyrazine ring and methoxyethyl chain point in the same direction as the side chain of the dioxonaphtho-imidazolium backbone (5l, $5 \mathbf{k}$, and $5 \mathbf{d}$ ), displayed $<30 \%$ of the activity of YM155 (Figure 7C). In addition, analogs adopting the same spatial conformation as YM155 but without aromatic rings exhibited low activity (5s). Electrostatic surface potential analysis revealed that the $3 \mathrm{D}$ structure and surface charge distribution of $5 \mathrm{~m}$ are the most similar to YM155 among all analogs (Figure 7C). SAR analysis using surface models suggested that YM155 analogs should maintain the aromatic ring and the hydrophobic chain in opposite orientations around the dioxonaphtho-imidazolium
A

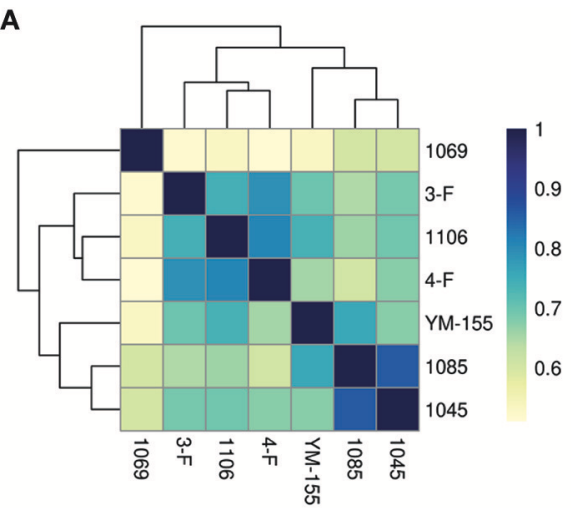

B

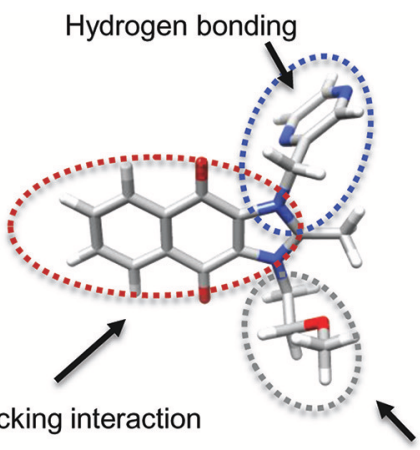

Hydrophobic interaction

C
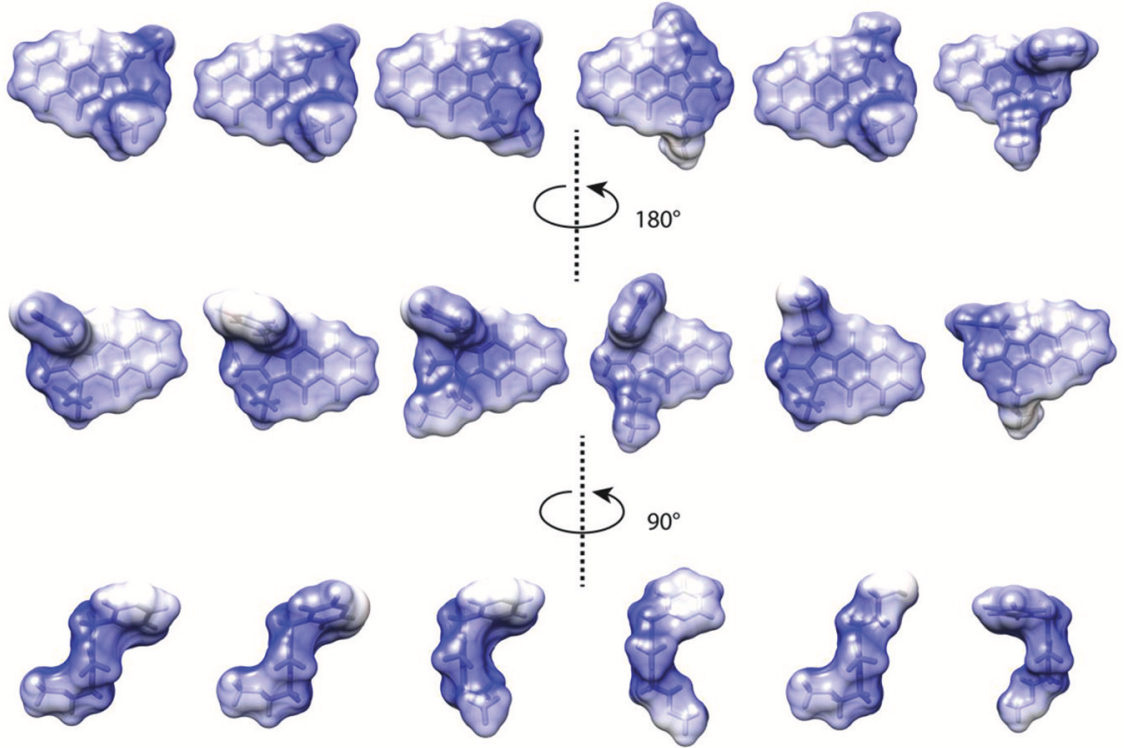
$90^{\circ}$
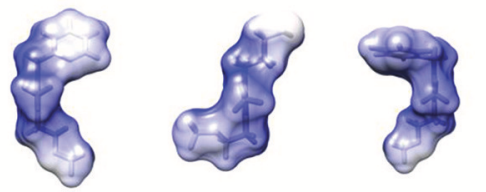

Activity
$(\%)$

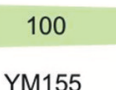

\begin{abstract}
$80-90$
\end{abstract}
$40-50$
51

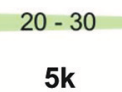

15 0

FIGURE 7 | Structure-activity relationships of YM155 analogs. (A) Correlation analysis of Tanimoto distance of YM155 analogs. (B) Energy minimized 3D structure of YM155 with stick representation method colored by gray (backbone), white (proton), nitrogen (blue), and red (oxygen), respectively. (C) Electrostatic surface potential of YM155 analogs was colored by blue (positively charged region) and red (negative charged region) with \pm 5 scaled energy kcal/(mole*e) unit. 
backbone to possess sufficient stemotoxic activity. Although the precise active site or pharmacophore to which YM155 analogs can bind remains unknown, the specific 3D (conformational) properties of YM155 analogs are likely to make a crucial contribution to interactions with the target protein, along with $\pi-\pi$ stacking, hydrogen bonding, and hydrophobic interactions.

\section{DISCUSSION}

As clinical trials of hPSC-based cell therapies are extended worldwide (Kimbrel and Lanza, 2015), associated risk factors, especially tumorigenicity, must be completely resolved to ensure safety (Heslop et al., 2015). Among various approaches for selective elimination of tumorigenic hPSCs (Jeong et al., 2017a), efficacy of teratoma inhibition, by relatively low dose of YM155 (at nM range), was determined by multiple independent studies (Bedel et al., 2017; Kang et al., 2017; Kim K. T. et al., 2017). Thus, SAR analysis of YM155 is important for developing more potent stemotoxic analogs. To this end, in the present work we synthesized 26 YM155 analogs with modified pyrazine ring structures, and found that the positions of hydrogen bond acceptors in the aromatic ring and the imidazolium ring are crucial for interactions with the target protein (possibly SLC35F2). The distance between the hydrogen bond acceptor and the imidazolium ring is one factor that is determinant of stemotoxic activity (Figure 2), and a nitrogen located in the meta or para position appears to be desirable (Figure 3, Figure S3). Similar results were obtained with $\mathbf{5 e}$ and $\mathbf{5 f}$, in which substitution of a fluoride with a sterically unfavorable methoxy group $(\mathbf{5} \mathbf{h}-\mathbf{5 j})$ resulted in the complete loss of activity (Figure 5) due to the high sensitivity to steric interactions. SLC35F2, a putative target protein of YM155, was shown to be responsible for uptake of YM155 in a cancer cell model (Winter et al., 2014). Because knockout of SLC35F2 in hESCs completely prevented hESC cell death by YM155 and its analogs (Figure 6), we speculate that hydrogen bonding interactions between YM155 and SLC35F2 may be critical for YM155 uptake, and consequentially for cytotoxic activity against both cancer cells and hPSCs. Since the 3D structure of SLC35F2 remains undetermined, precise determination of exactly how hydrogen bond acceptors on the aromatic ring structure may contribute to protein-ligand interactions cannot be achieved at present. For future application of YM155 and its analogs as both stemotoxic compounds and anti-cancer drugs (Nakahara et al., 2007; Clemens et al., 2015), it is important to determine the structure of SLC35F2 to facilitate the design of more potent

\section{REFERENCES}

Bedel, A., Beliveau, F., Lamrissi-Garcia, I., Rousseau, B., Moranvillier, I., Rucheton, B., et al. (2017). Preventing pluripotent cell teratoma in regenerative medicine applied to hematology disorders. Stem Cells Transl. Med. 6, 382-393. doi: 10.5966/sctm.2016-0201

Ben-David, U., and Benvenisty, N. (2011). The tumorigenicity of human embryonic and induced pluripotent stem cells. Nat. Rev. Cancer 11, 268-277. doi: $10.1038 / \operatorname{nrc} 3034$
YM155 analogs with increased uptake, and hence enhanced stemotoxic activity.

\section{CONCLUSION}

In summary, we synthesized total 26 YM155 analogs and demonstrated that nitrogen in the pyrazine ring can serve as a critical hydrogen bond acceptor, and these interactions are crucial for stemotoxic activity. Through structure-activity relationship (SAR) analysis, we developed a novel YM155 analog with a flurobenzyl group that has stemotoxic activity equivalent to that of YM-155. Additionally, we showed that the formation of hydrogen bonds involving the pyrazine ring structure is important for recognition of YM155 by SLC35F2, a membrane solute carrier protein, to induce cell death. Furthermore, analysis of the electrostatic surface potential supported the SAR evaluation of YM155 analogs based on 3D conformations.

\section{AUTHOR CONTRIBUTIONS}

CL and SC performed the chemical synthesis under supervision of Y-GS and S-HK. NMR, IR, LRMS, and HRMS were performed by CL and SC under supervision of Y-GS and S-HK. hPSCs and differentiated cell culture, Cell death assay, and Immunoblotting assay were performed by Y-HG and $\mathrm{H}-\mathrm{CJ}$ under the supervision of M-OL and H-JC. HL and WK performed Tanimoto analysis. Energy minimized 3D structure and electrostatic surface potential of YM155 analogs were generated by O-SK and WS. The manuscript was written by H-JC and S-HK with the support of WS, Y-GS, WK, and M-OL. All authors approved the manuscript in its final form for publication.

\section{FUNDING}

This research was funded by the National Research Foundation of Korea (NRF-2017M3A9B3061843: H-JC and NRF-2017R 1D1A1B03034612: S-HK), Seoul National University (370C20180086: H-JC), and the KRIBB Research Initiative Program (M-OL).

\section{SUPPLEMENTARY MATERIAL}

The Supplementary Material for this article can be found online at: https://www.frontiersin.org/articles/10.3389/fchem. 2019.00298/full\#supplementary-material
Ben-David, U., Gan, Q. F., Golan-Lev, T., Arora, P., Yanuka, O., Oren, Y. S., et al. (2013). Selective elimination of human pluripotent stem cells by an oleate synthesis inhibitor discovered in a high-throughput screen. Cell Stem Cell. 12, 167-179. doi: 10.1016/j.stem.2012.11.015

Blum, B., and Benvenisty, N. (2008). The tumorigenicity of human embryonic stem cells. Adv. Cancer Res. 100, 133-158. doi: 10.1016/S0065-230X(08)00005-5

Cho, S. J., Kim, K. T., Jeong, H. C., Park, J. C., Kwon, O. S., Song, Y. H., et al. (2018). Selective elimination of culture-adapted human embryonic stem cells with BH3 mimetics. Stem Cell Rep. 11, 1244-1256. doi: 10.1016/j.stemcr.2018.09.002 
Cho, S. J., Kim, S. Y., Jeong, H. C., Cheong, H., Kim, D., Park, S. J., et al. (2015). Repair of ischemic injury by pluripotent stem cell based cell therapy without teratoma through selective photosensitivity. Stem Cell Rep. 5, 1067-1080. doi: 10.1016/j.stemcr.2015.10.004

Cho, S. J., Kim, S. Y., Park, S. J., Song, N., Kwon, H. Y., Kang, N. Y., et al. (2016). Photodynamic approach for teratoma-free pluripotent stem cell therapy using CDyl and visible light. ACS Cent. Sci. 2, 604-607. doi: 10.1021/acscentsci.6b00099

Choo, A. B., Tan, H. L., Ang, S. N., Fong, W. J., Chin, A., Lo, J., et al. (2008). Selection against undifferentiated human embryonic stem cells by a cytotoxic antibody recognizing podocalyxin-like protein-1. Stem Cells. 26, 1454-1463. doi: 10.1634/stemcells.2007-0576

Clemens, M. R., Gladkov, O. A., Gartner, E., Vladimirov, V., Crown, J., Steinberg, J., et al. (2015). Phase II, multicenter, open-label, randomized study of YM155 plus docetaxel as first-line treatment in patients with HER2negative metastatic breast cancer. Breast Cancer Res. Treat. 149, 171-179. doi: 10.1007/s10549-014-3238-6

Goddard, T. D., Huang, C. C., and Ferrin, T. E. (2005). Software extensions to UCSF chimera for interactive visualization of large molecular assemblies. Structure 13, 473-482. doi: 10.1016/j.str.2005.01.006

Hanwell, M. D., Curtis, D. E., Lonie, D. C., Vandermeersch, T., Zurek, E., and Hutchison, G. R. (2012). Avogadro: an advanced semantic chemical editor, visualization, and analysis platform. J. Cheminform. 4:17. doi: 10.1186/1758-2946-4-17

Heslop, J. A., Hammond, T. G., Santeramo, I., Tort Piella, A., Hopp, I., Zhou, J., et al. (2015). Concise review: workshop review: understanding and assessing the risks of stem cell-based therapies. Stem Cells Transl. Med. 4, 389-400. doi: 10.5966/sctm.2014-0110

Ho, S. H., Ali, A., Ng, Y. C., Lam, K. K., Wang, S., Chan, W. K., et al. (2016). Dioxonaphthoimidazoliums are potent and selective rogue stem cell clearing agents with SOX2-suppressing properties. ChemMedChem 11, 1944-1955. doi: $10.1002 / \mathrm{cmdc} .201600262$

Ho, S. H., Sim, M. Y., Yee, W. L., Yang, T., Yuen, S. P., and Go, M. L. (2015). Antiproliferative, DNA intercalation and redox cycling activities of dioxonaphtho[2,3-d]imidazolium analogs of YM155: a structure-activity relationship study. Eur. J. Med. Chem. 104, 42-56. doi: 10.1016/j.ejmech.2015.09.026

Hong, M., Ren, M. Q., Silva, J., Paul, A., Wilson, W. D., Schroeder, C., et al. (2017). YM155 inhibits topoisomerase function. Anticancer. Drugs 28, 142-152. doi: 10.1097/CAD.0000000000000441

Jeong, H. C., Cho, S. J., Lee, M. O., and Cha, H. J. (2017a). Technical approaches to induce selective cell death of pluripotent stem cells. Cell. Mol. Life Sci. 74, 2601-2611. doi: 10.1007/s00018-017-2486-0

Jeong, H. C., Park, S. J., Choi, J. J., Go, Y. H., Hong, S. K., Kwon, O. S., et al. (2017b). PRMT8 controls the pluripotency and mesodermal fate of human embryonic stem cells by enhancing the PI3K/AKT/SOX2 axis. Stem Cells 35, 2037-2049. doi: $10.1002 /$ stem.2642

Kang, S. J., Park, Y. I., Hwang, S. R., Yi, H., Tham, N., Ku, H. O., et al. (2017). Hepatic population derived from human pluripotent stem cells is effectively increased by selective removal of undifferentiated stem cells using YM155. Stem Cell Res. Ther. 8:78. doi: 10.1186/s13287-017-0517-2

Kim, K.-T., Park, J.-C., Lee, H., Jang, H.-K., Jin, Y., Kim, W., et al. (2019). Scarless enriched selection of genome edited human pluripotent stem cells using induced drug resistance. BioRxiv [Preprint]. doi: 10.1101/522383

Kim, K. T., Jeong, H. C., Kim, C. Y., Kim, E. Y., Heo, S. H., Cho, S. J., et al. (2017). Intact wound repair activity of human mesenchymal stem cells after YM155 mediated selective ablation of undifferentiated human embryonic stem cells. J. Dermatol. Sci. 86, 123-131. doi: 10.1016/j.jdermsci.2017.01.011

Kim, S. Y., Jeong, H. C., Hong, S. K., Lee, M. O., Cho, S. J., and Cha, H. J. (2017). Quercetin induced ROS production triggers mitochondrial cell death of human embryonic stem cells. Oncotarget 8, 64964-64973. doi: 10.18632/oncotarget.11070

Kimbrel, E. A., and Lanza, R. (2015). Current status of pluripotent stem cells: moving the first therapies to the clinic. Nat. Rev. Drug Discov. 14, 681-692. doi: $10.1038 / \mathrm{nrd} 4738$

Kuo, S. C., Ibuka, T., Huang, L. J., Lien, J. C., Yean, S. R., Huang, S. C., et al. (1996). Synthesis and cytotoxicity of 1,2-disubstituted naphth[2,3-d]imidazole-4,9-diones and related compounds. J. Med. Chem. 39, 1447-1451. doi: 10.1021/jm950247k

Kwon, O.-S., Hong, S.-K., Kwon, S.-J., Go, Y.-H., Oh, E., and Cha, H.J. (2017). BCL2 induced by LAMTOR3/MAPK is a druggable target of chemoradioresistance in mesenchymal lung cancer. Cancer Lett. 403, 48-58. doi: 10.1016/j.canlet.2017.05.019

Lee, M. O., Moon, S. H., Jeong, H. C., Yi, J. Y., Lee, T. H., Shim, S. H., et al. (2013). Inhibition of pluripotent stem cell-derived teratoma formation by small molecules. Proc. Natl. Acad. Sci. U.S.A. 110, E3281-E3290. doi: $10.1073 /$ pnas. 1303669110

Lee, T. H., Song, S. H., Kim, K. L., Yi, J. Y., Shin, G. H., Kim, J. Y., et al. (2010). Functional recapitulation of smooth muscle cells via induced pluripotent stem cells from human aortic smooth muscle cells. Circ. Res. 106, 120-128. doi: 10.1161/CIRCRESAHA.109.207902

Maggiora, G., Vogt, M., Stumpfe, D., and Bajorath, J. (2014). Molecular similarity in medicinal chemistry. J. Med. Chem. 57, 3186-3204. doi: 10.1021/jm401411z

Mandai, M., Watanabe, A., Kurimoto, Y., Hirami, Y., Morinaga, C., Daimon, T., et al. (2017). Autologous induced stem-cell-derived retinal cells for macular degeneration. N. Engl. J. Med. 376, 1038-1046. doi: 10.1056/NEJMoa1608368

Nakahara, T., Kita, A., Yamanaka, K., Mori, M., Amino, N., Takeuchi, M., et al. (2007). YM155, a novel small-molecule survivin suppressant, induces regression of established human hormone-refractory prostate tumor xenografts. Cancer Res. 67, 8014-8021. doi: 10.1158/0008-5472.CAN-07-1343

Pettersen, E. F., Goddard, T. D., Huang, C. C., Couch, G. S., Greenblatt, D. M., Meng, E. C., et al. (2004). UCSF Chimera-a visualization system for exploratory research and analysis. J. Comput. Chem. 25, 1605-1612. doi: 10.1002/jcc.20084

Schwartz, S. D., Regillo, C. D., Lam, B. L., Eliott, D., Rosenfeld, P. J., Gregori, N. Z., et al. (2015). Human embryonic stem cell-derived retinal pigment epithelium in patients with age-related macular degeneration and Stargardt's macular dystrophy: follow-up of two open-label phase $1 / 2$ studies. Lancet $385,509-516$. doi: 10.1016/S0140-6736(14)61376-3

Sim, M. Y., Huynh, H., Go, M. L., and Yuen, J. S. P. (2017). Action of YM155 on clear cell renal cell carcinoma does not depend on survivin expression levels. PLoS ONE 12:e0178168. doi: 10.1371/journal.pone.0178168

Song, W. K., Park, K. M., Kim, H. J., Lee, J. H., Choi, J., Chong, S. Y., et al. (2015). Treatment of macular degeneration using embryonic stem cell-derived retinal pigment epithelium: preliminary results in Asian patients. Stem Cell Rep. 4, 860-872. doi: 10.1016/j.stemcr.2015.04.005

Trounson, A., and Dewitt, N. D. (2016). Pluripotent stem cells progressing to the clinic. Nat. Rev. Mol. Cell Biol. 17, 194-200. doi: 10.1038/nrm.2016.10

Wani, T. H., Surendran, S., Jana, A., Chakrabarty, A., and Chowdhury, G. (2018a). Quinone-based antitumor agent sepantronium bromide (YM155) causes oxygen-independent redox-activated oxidative DNA damage. Chem. Res. Toxicol. 31, 612-618. doi: 10.1021/acs.chemrestox.8b00094

Wani, T. H., Surendran, S., Mishra, V. S., Chaturvedi, J., Chowdhury, G., and Chakrabarty, A. (2018b). Adaptation to chronic exposure to sepantronium bromide (YM155), a prototypical survivin suppressant is due to persistent DNA damage-response in breast cancer cells. Oncotarget 9, 33589-33600. doi: 10.18632/oncotarget.26096

Winter, G. E., Radic, B., Mayor-Ruiz, C., Blomen, V. A., Trefzer, C., Kandasamy, R. K., et al. (2014). The solute carrier SLC35F2 enables YM155-mediated DNA damage toxicity. Nat. Chem. Biol. 10, 768-773. doi: 10.1038/nchembio.1590

Yagyu, S., Hoyos, V., Del Bufalo, F., and Brenner, M. K. (2015). An inducible Caspase-9 suicide gene to improve the safety of therapy using human induced pluripotent stem cells. Mol. Ther. 23, 1475-1485. doi: 10.1038/mt.2015.100

Conflict of Interest Statement: The authors declare that the research was conducted in the absence of any commercial or financial relationships that could be construed as a potential conflict of interest.

Copyright (C) 2019 Go, Lim, Jeong, Kwon, Chung, Lee, Kim, Suh, Son, Lee, Cha and Kim. This is an open-access article distributed under the terms of the Creative Commons Attribution License (CC BY). The use, distribution or reproduction in other forums is permitted, provided the original author(s) and the copyright owner(s) are credited and that the original publication in this journal is cited, in accordance with accepted academic practice. No use, distribution or reproduction is permitted which does not comply with these terms. 